\title{
Macronutrient and Major Food Group Intake in a Cohort of Southern Italian Adults
}

\author{
Serena Mulè ${ }^{1}$, Mariagiovanna Falla ${ }^{1}$, Alessandra Conti ${ }^{1}$, Dora Castiglione ${ }^{1}$, Isabella Blanco ${ }^{1}$ (D), \\ Armando Platania ${ }^{1}$, Maurizio D'Urso ${ }^{2}$ and Marina Marranzano ${ }^{1, *}$ \\ 1 Department of Medical and Surgical Sciences and Advanced Technologies "G.F. Ingrassia", \\ University of Catania, 95125 Catania, Italy; serenamule86@gmail.com (S.M.); \\ mariagiovannafalla@yahoo.com (M.F.); alessandra_conti@ymail.com (A.C.); \\ doracastiglione29@gmail.com (D.C.); dott.ssa.blanco.isabella@gmail.com (I.B.); armplt@hotmail.it (A.P.) \\ 2 Provincial Health Authority of Catania, 95127 Catania, Italy; ma.durso76@gmail.com \\ * Correspondence: marranz@unict.it; Tel.: +39-095-378-2180
}

Received: 11 March 2018; Accepted: 12 April 2018; Published: 15 April 2018

\begin{abstract}
Background: Dietary intake of macronutrient and foods is considered crucial to decrease the risk of diet-related non-communicable diseases. Methods: The aim of this study was to describe the intake of major food groups and macronutrients in a random sample of 1838 southern Italian adults. Results: No significant differences of macronutrient consumption between sexes were found. By contrast, younger individuals had significantly higher intake of animal protein than older ones. Men reported consuming significantly more total processed meats and less eggs than women; egg consumption significantly increased by age groups. Significantly lower intake of fruit in the younger age group compared to older ones was found. Various patterns of correlation between food groups were described. More than half of individuals reached the suggested recommendations for carbohydrate and fiber intake, and about two-thirds met the recommendations for total protein and cholesterol intake, while only a minority met for total fat intake. Total and plant protein, monounsaturated and omega-6 fatty acids, were significantly inversely related with BMI (body mass index), while trans fatty acids and cholesterol were directly correlated. A direct association with unprocessed meats and an inverse association with processed meats was also found. Conclusions: The overall findings suggest that relatively healthy dietary habits are common in southern Italy.
\end{abstract}

Keywords: macronutrients; food intake; body mass index; dietary recommendations; cohort

\section{Introduction}

Over the last decades, great efforts have been done to identify a nutritionally balanced diet that might help reduce the risk of chronic non-communicable diseases. There is convincing evidence that dietary factors, alongside with physical activity and abstinence from unhealthy lifestyle behaviors (such as smoking habits), play a crucial role in prolonging the lifespan and ameliorating human health [1,2]. Adequate nutritional requirements represent, nowadays, a key element of public health effort [3]; thus, assessment and knowledge of current populations' nutritional status is needed to design national recommendations [4]. Previous guidelines were mainly interested in macronutrient intake, but more recent dietary advice focused on food groups, in order to improve the understanding of the general population and facilitate public health educators and policymakers to better identify crucial priorities in the field [5,6].

Research in nutritional epidemiology produced over the last years investigated the association between macronutrients/major food groups, and the most common chronic non-communicable diseases [7]. As prevalence of metabolic disorders has increased in the last decades, major attention 
has been appointed to the risk of obesity, considered the potential lead mediating factor for many other conditions [8]. In contrast with the individual role of obesity as determinant of diet-related diseases, there is general agreement that calorie source matters, and that diet quality, intended as a proper ratio between macronutrients and individual food groups, constitutes an independent risk factor for negative outcomes [9]. As carbohydrates are generally the most common source of dietary energy, it is therefore intuitive to ascribe to them the major responsibility for higher risk of obesity. However, numerous studies failed in assessing such a relationship, making evidence on this matter difficult to understand [10]. In fact, whether carbohydrates come in the form of whole or refined grains, has been suggested to be relevant in the explanation for the uncertainty of the findings from the studies exploring the association between total carbohydrate intake and weight status [10]. Similar concerns regard dietary guidelines involving protein intake. In fact, there is adequate evidence (from randomized controlled trials, RCTs) showing that substitution of protein for carbohydrate may favorably affect weight management and improve cardiometabolic biomarkers [11,12]. However, the type of protein may have specific effects, and other studies reported that differences between animal and plant protein occur when exploring long-term association with metabolic disorders [13] and overall mortality risk $[14,15]$. Final important different effects have been recently associated with various dietary fats. The failure of "low-fat diets" in prolonging the lifespan [16] and the discovery of the beneficial effects of (relatively) "high-fat diets", such as the Mediterranean dietary pattern $[17,18]$, underlined the need to better distinguish between dietary fats and their effects on health. There is evidence that monoand polyunsaturated fatty acids (MUFA and PUFA, respectively), including omega-3 PUFA from fish and vegetable, may exert a number of beneficial effects compared to saturated or, even worse, trans-fatty acids $[19,20]$. However, evidence on unhealthy effects of saturated fatty acids, per se, is still controversial, and further research is needed, overall, to better distinguish between subgroups of macronutrients, as aforementioned.

National and international organizations are dealing with current evidence on the association between diet and health. Experts boards continuously draft and update dietary guidelines and recommendations in order to prevent, on a large population scale, common non-communicable diseases. However, data on actual food consumption in cohort studies is often underrated and scarcely described. The aim of the present study was to describe the intake of major food groups and macronutrients in a sample of southern Italian adults, and to analyze the differences in consumption between sexes and age groups. Additionally, the study aimed to explore the correlation between the variables investigated and the association with weight status of participants.

\section{Materials and Methods}

\subsection{Study Population}

A sample of 2044 men and women aged 18 or more was collected between 2014 and 2016 in the main districts of the city of Catania, southern Italy, to build the Mediterranean healthy eating, ageing, and lifestyle (MEAL) cohort. A detailed description of the study protocol is published elsewhere [21]. Briefly, the theoretical sample size was set at 1500 individuals to provide a specific relative precision of $5 \%$ (type I error, 0.05 ; type II error, 0.10 ), taking into account an anticipated $70 \%$ participation rate. The sampling technique included stratification by municipality area, age, and sex of inhabitants, and randomization into subgroups, with randomly selected general practitioners being the sampling units, and individuals registered to them comprising the final sample units. Out of 2405 individuals invited, the final sample size was 2044 participants (response rate of 85\%). All participants were informed about the aims of the study and provided a written informed consent. All the study procedures were carried out in accordance with the Declaration of Helsinki (1989) of the World Medical Association. The study protocol has been approved by the concerning ethical committee (protocol number: 802/23 December 2014). 


\subsection{Data Collection}

Data was collected by a face-to-face computer-assisted personal interview using tablet computers. In order to visualize the response options, participants were provided of a paper copy of the questionnaire, however, final answers were filled in by the interviewer directly on the digital device (tablet computer). The demographic and anthropometric data were collected according to standard procedures [22]. Regarding anthropometric measurements, height was measured to the nearest $0.5 \mathrm{~cm}$ without shoes, with the back square against the wall tape, eyes looking straight ahead, with a right-angle triangle resting on the scalp and against the wall. Weight was measured with a lever balance to the nearest $100 \mathrm{~g}$ without shoes and with light undergarments. Body mass index (BMI) was finally calculated [23].

\subsection{Dietary Assessment}

A food frequency questionnaire (FFQ) previously validated for the Sicilian population was administered to collect information on food consumption [24,25]. The long version of the FFQ used to retrieve the dietary estimates presented in this study consisted of 110 food items; intake of seasonal foods referred to consumption during the period in which the food was available, and then adjusted by its proportional intake in one year. Following the identification of the food frequency consumption, the estimated intakes were converted into daily intake (g/day) and were used to calculate energy and macronutrient content based on online food composition databases (such as the Research Center for Foods and Nutrition CREA-Consiglio per la ricerca in agricoltura e l'analisi dell'economia agraria) [26]. Nutrient intake was finally adjusted for total energy intake (kcal/day) using the residual method [27]. FFQs with unreliable intakes (we arbitrarily considered $<1000$ or $>6000 \mathrm{kcal} /$ day as realistically unreliable energy intake; $n=107)$ as well as missing items for the purposes of this study $(n=99)$ were excluded from the analyses, leaving a total of 1838 individuals included in the analysis.

\subsection{Dietary Recommendations}

To investigate agreement with dietary recommendations, we used the European proposed values for macronutrient intake of the European Food Safety Agency (EFSA) [28] and those proposed by the Italian Society of Human Nutrition "Livelli di Assunzione di Riferimento di Nutrienti" (LARN) [29], while for major food groups we used the World Health Organization (WHO) recommendations [30].

\subsection{Statistical Analysis}

Frequencies are presented as absolute numbers and percentages; continuous variables are presented as means and standard errors, medians and ranges. Differences between groups for continuous variables were compared with Student's $t$ test and ANOVA for continuous variables distributed normally, and Mann-Whitney U test and Kruskal-Wallis test for variables not normally distributed. Correlations among major food groups were tested through calculation of Pearson's or Spearman's correlation coefficients, depending on the distribution of the variable. Linear association between variables of interest and BMI levels were tested through linear regression analyses. All reported $P$ values were based on two-sided tests and compared to a significance level of $5 \%$. SPSS 17 (SPSS Inc., Chicago, IL, USA) software was used for all the statistical calculations.

\section{Results}

Table 1 shows the distribution of total energy, macronutrient and fiber intake in the study cohort, by sex and age groups. No significant differences of mean consumption of macronutrients between sexes were found. All macronutrients were mostly equally distributed, and even though men had slightly higher intake of cholesterol and total protein, the difference was not significant compared to women. In contrast, younger individuals consumed significantly more animal protein than older ones. 
The description of consumption of major food group from animal source is shown in Table 2. Regarding differences between sex, men reported consuming significantly more total processed meats and less eggs than women (18.00 g/day vs. $14.54 \mathrm{~g} /$ day and $2.04 \mathrm{~g} /$ day vs. $2.62 \mathrm{~g} /$ day, respectively). Difference in egg consumption was also found between age groups, as intake significantly increased with age. Table 3 describes distribution of intake of plant food groups between sex and age groups. No significant differences were evident between sexes, but a significantly lower intake of fruit in the younger age group compared to older ones was found.

The correlation between intake of all major food groups is shown in Table 4. A correlation between fruit, vegetables, legumes, and seafood was found; however, the latter were also correlated with all other animal products, including cheese, eggs, and processed and unprocessed red meats. Whole and refined grain intake was correlated with yoghurt, while nuts and seeds were correlated with both meat and vegetable product intake. However, most of the significant associations were very weak and arguably negligible.

Table 5 describes the percentage of individuals meeting recommendations from LARN, EFSA, and WHO on macronutrients and food group intake. Generally, more than half of individuals reached the suggested recommendations for carbohydrate and fiber intake, while the proportion of adherent individuals was even higher for total protein and cholesterol intake recommendations. By contrast, only a minority met the recommendations for total fat intake.

Tables 6 and 7 describe the association between macronutrient and major food group intake and BMI levels in the investigated population, total and by sex. Total protein, and specifically plant protein, monounsaturated fatty acids and omega- 6 fatty acids were significantly inversely related with BMI, while trans fatty acids and cholesterol were directly correlated (Table 6). However, no significant results were found for major food groups, with the exception of a direct association with unprocessed meats and an inverse association with processed meats (Table 7). It was noteworthy that the magnitude of the latter associations and of proteins, in general, were very small compared to those of dietary fats. 
Table 1. Total, sex, and age group-specific consumption of macronutrients and fiber in the study participants of the Mediterranean healthy eating, ageing, and lifestyle (MEAL) study $(n=1838)$. ${ }^{*}$ denotes $p<0.05$.

\begin{tabular}{|c|c|c|c|c|c|c|c|c|c|c|c|c|c|c|c|}
\hline & \multicolumn{3}{|c|}{ Total } & \multicolumn{3}{|c|}{$<20$ years } & \multicolumn{3}{|c|}{$20<$ years $<50$} & \multicolumn{3}{|c|}{$50<$ years $<70$} & \multicolumn{3}{|c|}{$>70$ years } \\
\hline & $n$ & $\begin{array}{l}\text { Mean } \\
\text { (SE) }\end{array}$ & $\begin{array}{l}\text { Median } \\
\text { (Range) }\end{array}$ & $n$ & $\begin{array}{c}\text { Mean } \\
\text { (SE) }\end{array}$ & $\begin{array}{l}\text { Median } \\
\text { (Range) }\end{array}$ & $n$ & $\begin{array}{c}\text { Mean } \\
\text { (SE) }\end{array}$ & $\begin{array}{l}\text { Median } \\
\text { (Range) }\end{array}$ & $n$ & $\begin{array}{c}\text { Mean } \\
\text { (SE) }\end{array}$ & $\begin{array}{l}\text { Median } \\
\text { (Range) }\end{array}$ & $n$ & $\begin{array}{c}\text { Mean } \\
\text { (SE) }\end{array}$ & $\begin{array}{l}\text { Median } \\
\text { (Range) }\end{array}$ \\
\hline \multicolumn{16}{|c|}{ Total Energy } \\
\hline Total & 1838 & $\begin{array}{c}2022.80 \\
(15.30)\end{array}$ & $\begin{array}{l}1927.63 \\
(1000.80 \\
4974.01)\end{array}$ & 53 & $\begin{array}{c}2037.36 \\
(79.24)\end{array}$ & $\begin{array}{c}1986.05 \\
(1025.75 \\
3728.68)\end{array}$ & 963 & $\begin{array}{c}2027.51 \\
(21.91)\end{array}$ & $\begin{array}{l}1930.64 \\
(1000.80 \\
4865.38)\end{array}$ & 597 & $\begin{array}{c}2038.93 \\
(27.00)\end{array}$ & $\begin{array}{l}1920.70 \\
(1015.33 \\
4974.01)\end{array}$ & 225 & $\begin{array}{c}1956.43 \\
(36.66)\end{array}$ & $\begin{array}{c}1923.88 \\
(1010.31 \\
3379.32)\end{array}$ \\
\hline Men & 772 & $\begin{array}{c}2054.16 \\
(25.38)\end{array}$ & $\begin{array}{c}1939.18 \\
(1012.84 \\
4974.01)\end{array}$ & 30 & $\begin{array}{l}2101.73 \\
(111.24)\end{array}$ & $\begin{array}{c}2048.38 \\
(1025.75 \\
3728.68)\end{array}$ & 384 & $\begin{array}{c}2047.09 \\
(37.99)\end{array}$ & $\begin{array}{l}1907.56 \\
(1012.84 \\
4865.38)\end{array}$ & 265 & $\begin{array}{c}2076.45 \\
(43.57)\end{array}$ & $\begin{array}{c}1938.57 \\
(1015.33, \\
4974.01)\end{array}$ & 93 & $\begin{array}{c}2004.48 \\
(56.27)\end{array}$ & $\begin{array}{c}2025.94 \\
(1019.88, \\
3334.93)\end{array}$ \\
\hline Women & 1066 & $\begin{array}{c}2000.09 \\
(18.90)\end{array}$ & $\begin{array}{c}1915.60 \\
(1000.80 \\
3915.46)\end{array}$ & 23 & $\begin{array}{c}1953.41 \\
(111.15)\end{array}$ & $\begin{array}{c}1873.20 \\
(1133.85 \\
3547.77)\end{array}$ & 579 & $\begin{array}{c}2014.52 \\
(26.35)\end{array}$ & $\begin{array}{c}1942.98 \\
(1000.80 \\
3915,46)\end{array}$ & 332 & $\begin{array}{c}2008.99 \\
(33.84)\end{array}$ & $\begin{array}{l}1902.58 \\
(1016.84 \\
3911.26)\end{array}$ & 132 & $\begin{array}{c}1922.58 \\
(48.27)\end{array}$ & $\begin{array}{c}1887.88 \\
(1010.31 \\
3379.32)\end{array}$ \\
\hline \multicolumn{16}{|c|}{ Saturated fat } \\
\hline Total & 1838 & $\begin{array}{l}23.58 \\
(0.23)\end{array}$ & $\begin{array}{l}22.17 \\
(6.49 \\
80.45) \\
\end{array}$ & 53 & $\begin{array}{l}25.55 \\
(1.29)\end{array}$ & $\begin{array}{c}26.51 \\
(11.85, \\
55.77)\end{array}$ & 963 & $\begin{array}{l}23.71 \\
(0.33)\end{array}$ & $\begin{array}{l}22.11 \\
(6.49 \\
80.45)\end{array}$ & 597 & $\begin{array}{l}23.52 \\
(0.41)\end{array}$ & $\begin{array}{l}21.88 \\
(6.91 \\
74.26)\end{array}$ & 225 & $\begin{array}{l}22.69 \\
(0.55)\end{array}$ & $\begin{array}{l}22.87 \\
(6.60 \\
45.05)\end{array}$ \\
\hline Men & 772 & $\begin{array}{l}23.86 \\
(0.38)\end{array}$ & $\begin{array}{l}22.09 \\
(6.60 \\
80.45) \\
\end{array}$ & 30 & $\begin{array}{l}27.89 \\
(1.80)\end{array}$ & $\begin{array}{l}26.80 \\
(14.82, \\
55.77) \\
\end{array}$ & 384 & $\begin{array}{l}23.45 \\
(0.55)\end{array}$ & $\begin{array}{l}20.88 \\
(8.61 \\
80.45) \\
\end{array}$ & 265 & $\begin{array}{l}24.04 \\
(0.64)\end{array}$ & $\begin{array}{l}22.20 \\
(9.47 \\
74.26) \\
\end{array}$ & 93 & $\begin{array}{l}23.75 \\
(0.88)\end{array}$ & $\begin{array}{l}24.47 \\
(6.60 \\
44.82)\end{array}$ \\
\hline Women & 1066 & $\begin{array}{l}23.37 \\
(0.29)\end{array}$ & $\begin{array}{l}22.33 \\
(6.49 \\
62.82)\end{array}$ & 23 & $\begin{array}{l}22.50 \\
(1.64)\end{array}$ & $\begin{array}{l}21.09 \\
(11.85 \\
34.77)\end{array}$ & 579 & $\begin{array}{l}23.88 \\
(0.40)\end{array}$ & $\begin{array}{l}23.00 \\
(6.49 \\
62.82)\end{array}$ & 332 & $\begin{array}{l}23.11 \\
(0.52)\end{array}$ & $\begin{array}{l}21.46 \\
(6.91 \\
61.79)\end{array}$ & 132 & $\begin{array}{l}21.94 \\
(0.69)\end{array}$ & $\begin{array}{l}21.65 \\
(6.97 \\
45.05)\end{array}$ \\
\hline
\end{tabular}


Table 1. Cont.

\begin{tabular}{|c|c|c|c|c|c|c|c|c|c|c|c|c|c|c|c|}
\hline & \multicolumn{3}{|c|}{ Total } & \multicolumn{3}{|c|}{$<20$ years } & \multicolumn{3}{|c|}{$20<$ years $<50$} & \multicolumn{3}{|c|}{$50<$ years $<70$} & \multicolumn{3}{|c|}{$>70$ years } \\
\hline & $n$ & $\begin{array}{c}\text { Mean } \\
\text { (SE) }\end{array}$ & $\begin{array}{l}\text { Median } \\
\text { (Range) }\end{array}$ & $n$ & $\begin{array}{l}\text { Mean } \\
\text { (SE) }\end{array}$ & $\begin{array}{l}\text { Median } \\
\text { (Range) }\end{array}$ & $n$ & $\begin{array}{l}\text { Mean } \\
\text { (SE) }\end{array}$ & $\begin{array}{l}\text { Median } \\
\text { (Range) }\end{array}$ & $n$ & $\begin{array}{c}\text { Mean } \\
\text { (SE) }\end{array}$ & $\begin{array}{l}\text { Median } \\
\text { (Range) }\end{array}$ & $n$ & $\begin{array}{c}\text { Mean } \\
\text { (SE) }\end{array}$ & $\begin{array}{l}\text { Median } \\
\text { (Range) }\end{array}$ \\
\hline \multicolumn{16}{|c|}{$\begin{array}{l}\text { Monounsaturated } \\
\text { fat }\end{array}$} \\
\hline Total & 1838 & $\begin{array}{l}25.30 \\
(0.21)\end{array}$ & $\begin{array}{l}24.13 \\
(7.29 \\
93.85)\end{array}$ & 53 & $\begin{array}{l}27.41 \\
(1.23)\end{array}$ & $\begin{array}{l}25.73 \\
(13.19, \\
63.32)\end{array}$ & 963 & $\begin{array}{l}25.39 \\
(0.29)\end{array}$ & $\begin{array}{l}24.04 \\
(7.29 \\
80.32)\end{array}$ & 597 & $\begin{array}{l}25.28 \\
(0.38)\end{array}$ & $\begin{array}{l}23.99 \\
(11.01, \\
93.85)\end{array}$ & 225 & $\begin{array}{l}24.48 \\
(0.49)\end{array}$ & $\begin{array}{l}24.07 \\
(7.61, \\
47.14)\end{array}$ \\
\hline Men & 772 & $\begin{array}{l}25.62 \\
(0.36)\end{array}$ & $\begin{array}{l}23.94 \\
(7.61, \\
93.85)\end{array}$ & 30 & $\begin{array}{l}29.12 \\
(1.83)\end{array}$ & $\begin{array}{l}26.56 \\
(15.20, \\
63.32)\end{array}$ & 384 & $\begin{array}{l}25.12 \\
(0.51)\end{array}$ & $\begin{array}{l}23.19 \\
(11.71, \\
80.32)\end{array}$ & 265 & $\begin{array}{l}25.98 \\
(0.66)\end{array}$ & $\begin{array}{l}24.26 \\
(11.77, \\
93.85)\end{array}$ & 93 & $\begin{array}{l}25.56 \\
(0.80)\end{array}$ & $\begin{array}{l}26.43 \\
(7.61, \\
47.14)\end{array}$ \\
\hline Women & 1066 & $\begin{array}{l}25.07 \\
(0.25)\end{array}$ & $\begin{array}{l}24.19 \\
(7.29 \\
62.38)\end{array}$ & 23 & $\begin{array}{l}25.18 \\
(1.45)\end{array}$ & $\begin{array}{l}24.10 \\
(13.19, \\
41.50)\end{array}$ & 579 & $\begin{array}{l}25.57 \\
(0.35)\end{array}$ & $\begin{array}{l}24.74 \\
(7.29 \\
62.38)\end{array}$ & 332 & $\begin{array}{l}24.72 \\
(0.43)\end{array}$ & $\begin{array}{l}23.67 \\
(11.01, \\
55.05)\end{array}$ & 132 & $\begin{array}{l}23.71 \\
(0.60)\end{array}$ & $\begin{array}{c}23.04 \\
(11.03, \\
44.77)\end{array}$ \\
\hline \multicolumn{16}{|l|}{$\begin{array}{c}\text { Total } \\
\text { omega-6 } \\
\text { fatty acids }\end{array}$} \\
\hline Total & 1838 & $\begin{array}{c}9.92 \\
(0.10)\end{array}$ & $\begin{array}{c}9.21 \\
(3.08 \\
55.51)\end{array}$ & 53 & $\begin{array}{c}10.33 \\
(0.61)\end{array}$ & $\begin{array}{c}8.94 \\
(3.36 \\
27.60)\end{array}$ & 963 & $\begin{array}{c}9.97 \\
(0.14)\end{array}$ & $\begin{array}{c}9.21 \\
(3.08 \\
30.64)\end{array}$ & 597 & $\begin{array}{l}10.03 \\
(0.19)\end{array}$ & $\begin{array}{c}9.22 \\
(3.29 \\
55.51)\end{array}$ & 225 & $\begin{array}{c}9.29 \\
(0.21)\end{array}$ & $\begin{array}{c}9.13 \\
(3.64 \\
20.44)\end{array}$ \\
\hline Men & 772 & $\begin{array}{c}9.99 \\
(0.16)\end{array}$ & $\begin{array}{r}9.09 \\
(3.08 \\
55.51)\end{array}$ & 30 & $\begin{array}{l}10.49 \\
(0.86)\end{array}$ & $\begin{array}{c}9.43 \\
(3.36 \\
27.60)\end{array}$ & 384 & $\begin{array}{c}9.93 \\
(0.23)\end{array}$ & $\begin{array}{c}8.96 \\
(3.08 \\
29.06)\end{array}$ & 265 & $\begin{array}{l}10.19 \\
(0.31)\end{array}$ & $\begin{array}{c}9.18 \\
(3.29 \\
55.51)\end{array}$ & 93 & $\begin{array}{c}9.54 \\
(0.30)\end{array}$ & $\begin{array}{c}9.43 \\
(4.01 \\
16.09)\end{array}$ \\
\hline Women & 1066 & $\begin{array}{c}9.87 \\
(0.12)\end{array}$ & $\begin{array}{r}9.27 \\
(3.61 \\
32.07)\end{array}$ & 23 & $\begin{array}{c}10.11 \\
(0.89)\end{array}$ & $\begin{array}{c}8.92 \\
(4.89 \\
23.60)\end{array}$ & 579 & $\begin{array}{l}10.00 \\
(0.17)\end{array}$ & $\begin{array}{c}9.37 \\
(3.61 \\
30.64)\end{array}$ & 332 & $\begin{array}{c}9.91 \\
(0.23)\end{array}$ & $\begin{array}{c}9.24 \\
(3.69 \\
32.07)\end{array}$ & 132 & $\begin{array}{c}9.12 \\
(0.29)\end{array}$ & $\begin{array}{c}9.03 \\
(3.64 \\
20.44)\end{array}$ \\
\hline
\end{tabular}


Table 1. Cont.

\begin{tabular}{|c|c|c|c|c|c|c|c|c|c|c|c|c|c|c|c|}
\hline & \multicolumn{3}{|c|}{ Total } & \multicolumn{3}{|c|}{$<20$ years } & \multicolumn{3}{|c|}{$20<$ years $<50$} & \multicolumn{3}{|c|}{$50<$ years $<70$} & \multicolumn{3}{|c|}{$>70$ years } \\
\hline & $n$ & $\begin{array}{c}\text { Mean } \\
\text { (SE) }\end{array}$ & $\begin{array}{l}\text { Median } \\
\text { (Range) }\end{array}$ & $n$ & $\begin{array}{c}\text { Mean } \\
\text { (SE) }\end{array}$ & $\begin{array}{l}\text { Median } \\
\text { (Range) }\end{array}$ & $n$ & $\begin{array}{c}\text { Mean } \\
\text { (SE) }\end{array}$ & $\begin{array}{l}\text { Median } \\
\text { (Range) }\end{array}$ & $n$ & $\begin{array}{c}\text { Mean } \\
\text { (SE) }\end{array}$ & $\begin{array}{l}\text { Median } \\
\text { (Range) }\end{array}$ & $n$ & $\begin{array}{c}\text { Mean } \\
\text { (SE) }\end{array}$ & $\begin{array}{l}\text { Median } \\
\text { (Range) }\end{array}$ \\
\hline \multicolumn{16}{|c|}{$\begin{array}{c}\text { Seafood } \\
\text { omega-3 fat }\end{array}$} \\
\hline Total & 1838 & $\begin{array}{c}0.53 \\
(0.01)\end{array}$ & $\begin{array}{c}0.38 \\
(0.00 \\
5.24)\end{array}$ & 53 & $\begin{array}{c}0.55 \\
(0.05)\end{array}$ & $\begin{array}{c}0.42 \\
(0.00 \\
1.56)\end{array}$ & 963 & $\begin{array}{c}0.52 \\
(0.02)\end{array}$ & $\begin{array}{c}0.36 \\
(0.00 \\
5.24)\end{array}$ & 597 & $\begin{array}{c}0.56 \\
(0.02)\end{array}$ & $\begin{array}{c}0.41 \\
(0.01 \\
5.06)\end{array}$ & 225 & $\begin{array}{c}0.53 \\
(0.03)\end{array}$ & $\begin{array}{c}0.43 \\
(0.03 \\
3.26)\end{array}$ \\
\hline Men & 772 & $\begin{array}{c}0.53 \\
(0.02)\end{array}$ & $\begin{array}{c}0.38 \\
(0.00 \\
5.24)\end{array}$ & 30 & $\begin{array}{c}0.49 \\
(0.07)\end{array}$ & $\begin{array}{c}0.36 \\
(0.00 \\
1.34)\end{array}$ & 384 & $\begin{array}{c}0.50 \\
(0.03)\end{array}$ & $\begin{array}{c}0.34 \\
(0.00 \\
5.24)\end{array}$ & 265 & $\begin{array}{c}0.58 \\
(0.04)\end{array}$ & $\begin{array}{c}0.44 \\
(0.05 \\
5.06)\end{array}$ & 93 & $\begin{array}{c}0.56 \\
(0.06)\end{array}$ & $\begin{array}{c}0.42 \\
(0.05 \\
3.26)\end{array}$ \\
\hline Women & 1066 & $\begin{array}{c}0.53 \\
(0.02)\end{array}$ & $\begin{array}{c}0.39 \\
(0.00 \\
3.97)\end{array}$ & 23 & $\begin{array}{c}0.63 \\
(0.08)\end{array}$ & $\begin{array}{c}0.56 \\
(0.17 \\
1.56) \\
\end{array}$ & 579 & $\begin{array}{c}0.53 \\
(0.02)\end{array}$ & $\begin{array}{c}0.38 \\
(0.00 \\
3.97)\end{array}$ & 332 & $\begin{array}{c}0.54 \\
(0.03)\end{array}$ & $\begin{array}{c}0.38 \\
(0.01 \\
3.05)\end{array}$ & 132 & $\begin{array}{c}0.51 \\
(0.04)\end{array}$ & $\begin{array}{c}0.43 \\
(0.03 \\
2.98)\end{array}$ \\
\hline \multicolumn{16}{|c|}{$\begin{array}{c}\text { Plant } \\
\text { omega-3 fat }\end{array}$} \\
\hline Total & 1838 & $\begin{array}{c}1.17 \\
(0.01)\end{array}$ & $\begin{array}{c}1.06 \\
(0.39 \\
5.66)\end{array}$ & 53 & $\begin{array}{c}1.23 \\
(0.08)\end{array}$ & $\begin{array}{c}1.08 \\
(0.51 \\
3.78)\end{array}$ & 963 & $\begin{array}{c}1.17 \\
(0.02)\end{array}$ & $\begin{array}{c}1.06 \\
(0.41 \\
5.51)\end{array}$ & 597 & $\begin{array}{c}1.20 \\
(0.02)\end{array}$ & $\begin{array}{c}1.06 \\
(0.42, \\
5.66)\end{array}$ & 225 & $\begin{array}{c}1.10 \\
(0.03)\end{array}$ & $\begin{array}{c}1.05 \\
(0.39 \\
2.79) \\
\end{array}$ \\
\hline Men & 772 & $\begin{array}{c}1.17 \\
(0.02)\end{array}$ & $\begin{array}{c}1.06 \\
(0.41 \\
4.48)\end{array}$ & 30 & $\begin{array}{c}1.25 \\
(0.11)\end{array}$ & $\begin{array}{c}1.09 \\
(0.60 \\
3.78)\end{array}$ & 384 & $\begin{array}{c}1.17 \\
(0.03)\end{array}$ & $\begin{array}{c}1.02 \\
(0.41 \\
3.93)\end{array}$ & 265 & $\begin{array}{c}1.18 \\
(0.03)\end{array}$ & $\begin{array}{c}1.07 \\
(0.46 \\
4.48)\end{array}$ & 93 & $\begin{array}{c}1.14 \\
(0.04)\end{array}$ & $\begin{array}{c}1.13 \\
(0.46 \\
2.32)\end{array}$ \\
\hline Women & 1066 & $\begin{array}{c}1.18 \\
(0.02)\end{array}$ & $\begin{array}{c}1.06 \\
(0.39 \\
5.66)\end{array}$ & 23 & $\begin{array}{c}1.20 \\
(0.12)\end{array}$ & $\begin{array}{c}0.90 \\
(0.51 \\
2.82)\end{array}$ & 579 & $\begin{array}{c}1.18 \\
(0.02)\end{array}$ & $\begin{array}{c}1.09 \\
(0.42, \\
5.51)\end{array}$ & 332 & $\begin{array}{c}1.21 \\
(0.03)\end{array}$ & $\begin{array}{c}1.05 \\
(0.42, \\
5.66)\end{array}$ & 132 & $\begin{array}{c}1.08 \\
(0.04)\end{array}$ & $\begin{array}{c}1.02 \\
(0.39 \\
2.79)\end{array}$ \\
\hline
\end{tabular}


Table 1. Cont.

\begin{tabular}{|c|c|c|c|c|c|c|c|c|c|c|c|c|c|c|c|}
\hline & \multicolumn{3}{|c|}{ Total } & \multicolumn{3}{|c|}{$<20$ years } & \multicolumn{3}{|c|}{$20<$ years $<50$} & \multicolumn{3}{|c|}{$50<$ years $<70$} & \multicolumn{3}{|c|}{$>70$ years } \\
\hline & $n$ & $\begin{array}{c}\text { Mean } \\
\text { (SE) }\end{array}$ & $\begin{array}{l}\text { Median } \\
\text { (Range) }\end{array}$ & $n$ & $\begin{array}{c}\text { Mean } \\
\text { (SE) }\end{array}$ & $\begin{array}{l}\text { Median } \\
\text { (Range) }\end{array}$ & $n$ & $\begin{array}{c}\text { Mean } \\
\text { (SE) }\end{array}$ & $\begin{array}{l}\text { Median } \\
\text { (Range) }\end{array}$ & $n$ & $\begin{array}{c}\text { Mean } \\
\text { (SE) }\end{array}$ & $\begin{array}{l}\text { Median } \\
\text { (Range) }\end{array}$ & $n$ & $\begin{array}{c}\text { Mean } \\
\text { (SE) }\end{array}$ & $\begin{array}{l}\text { Median } \\
\text { (Range) }\end{array}$ \\
\hline \multicolumn{16}{|c|}{$\begin{array}{c}\text { Trans fatty } \\
\text { acid }\end{array}$} \\
\hline Total & 1838 & $\begin{array}{l}32.31 \\
(0.28)\end{array}$ & $\begin{array}{r}30.83 \\
(10.30 \\
135.12)\end{array}$ & 53 & $\begin{array}{l}34.60 \\
(1.69)\end{array}$ & $\begin{array}{c}31.68 \\
(16.68, \\
84.81) \\
\end{array}$ & 963 & $\begin{array}{l}32.38 \\
(0.39)\end{array}$ & $\begin{array}{r}30.82 \\
(10.30 \\
100.42)\end{array}$ & 597 & $\begin{array}{l}32.46 \\
(0.50)\end{array}$ & $\begin{array}{r}31.02 \\
(12.26 \\
135.12)\end{array}$ & 225 & $\begin{array}{l}31.09 \\
(0.62)\end{array}$ & $\begin{array}{c}30.07 \\
(10.99 \\
59.82) \\
\end{array}$ \\
\hline Men & 772 & $\begin{array}{l}32.63 \\
(0.47)\end{array}$ & $\begin{array}{r}30.63 \\
(10.99 \\
135.12)\end{array}$ & 30 & $\begin{array}{l}36.18 \\
(2.50)\end{array}$ & $\begin{array}{l}32.79 \\
(17.03, \\
84.81)\end{array}$ & 384 & $\begin{array}{l}32.05 \\
(0.66)\end{array}$ & $\begin{array}{c}29.66 \\
(11.97 \\
100.42)\end{array}$ & 265 & $\begin{array}{l}33.19 \\
(0.88)\end{array}$ & $\begin{array}{r}31.42 \\
(12.26 \\
135.12)\end{array}$ & 93 & $\begin{array}{l}32.26 \\
(1.00)\end{array}$ & $\begin{array}{c}32.83 \\
(10.99 \\
59.82)\end{array}$ \\
\hline Women & 1066 & $\begin{array}{l}32.08 \\
(0.33)\end{array}$ & $\begin{array}{c}30.87 \\
(10.30, \\
84.75)\end{array}$ & 23 & $\begin{array}{l}32.55 \\
(2.12)\end{array}$ & $\begin{array}{c}30.14 \\
(16.68 \\
59.93) \\
\end{array}$ & 579 & $\begin{array}{l}32.60 \\
(0.47)\end{array}$ & $\begin{array}{l}31.38 \\
(10.30 \\
84.75) \\
\end{array}$ & 332 & $\begin{array}{l}31.88 \\
(0.58)\end{array}$ & $\begin{array}{c}30.35 \\
(14.88 \\
68.99) \\
\end{array}$ & 132 & $\begin{array}{l}30.28 \\
(0.78)\end{array}$ & $\begin{array}{l}29.62 \\
(13.84 \\
55.51)\end{array}$ \\
\hline \multicolumn{16}{|c|}{$\begin{array}{c}\text { Dietary } \\
\text { cholesterol }\end{array}$} \\
\hline Total & 1838 & $\begin{array}{c}187.55 \\
(1.93)\end{array}$ & $\begin{array}{c}175.00 \\
(17.29 \\
921.07)\end{array}$ & 53 & $\begin{array}{c}198.15 \\
(9.17)\end{array}$ & $\begin{array}{c}191.00 \\
(87.71 \\
371.79) \\
\end{array}$ & 963 & $\begin{array}{l}187.15 \\
(2.74)\end{array}$ & $\begin{array}{l}173.89 \\
(17.29 \\
921.07)\end{array}$ & 597 & $\begin{array}{c}188.24 \\
(3.44)\end{array}$ & $\begin{array}{r}172.62 \\
(59.94 \\
876.81) \\
\end{array}$ & 225 & $\begin{array}{c}184.84 \\
(4.92)\end{array}$ & $\begin{array}{l}180.36 \\
(42.92 \\
521.31) \\
\end{array}$ \\
\hline Men & 772 & $\begin{array}{l}191.35 \\
(3.22)\end{array}$ & $\begin{array}{l}174.09 \\
(42.92, \\
921.07)\end{array}$ & 30 & $\begin{array}{l}206.26 \\
(12.70)\end{array}$ & $\begin{array}{c}206.42 \\
(102.85 \\
371.79)\end{array}$ & 384 & $\begin{array}{l}186.61 \\
(4.64)\end{array}$ & $\begin{array}{c}164.24 \\
(56.99 \\
921.07)\end{array}$ & 265 & $\begin{array}{c}195.15 \\
(5.78)\end{array}$ & $\begin{array}{r}176.70 \\
(63.53 \\
876.81)\end{array}$ & 93 & $\begin{array}{c}195.29 \\
(7.83)\end{array}$ & $\begin{array}{l}189.94 \\
(42.92 \\
521.31)\end{array}$ \\
\hline Women & 1066 & $\begin{array}{l}184.80 \\
(2.37)\end{array}$ & $\begin{array}{c}176.30 \\
(17.29 \\
594.94)\end{array}$ & 23 & $\begin{array}{l}187.57 \\
(13.09)\end{array}$ & $\begin{array}{l}177.00 \\
(87.71 \\
333.42)\end{array}$ & 579 & $\begin{array}{l}187.51 \\
(3.35)\end{array}$ & $\begin{array}{l}181.67 \\
(17.29 \\
594.94)\end{array}$ & 332 & $\begin{array}{c}182.73 \\
(4.10)\end{array}$ & $\begin{array}{l}164.69 \\
(59.94 \\
487.38)\end{array}$ & 132 & $\begin{array}{l}177.65 \\
(6.26)\end{array}$ & $\begin{array}{l}169.40 \\
(56.35, \\
475.25)\end{array}$ \\
\hline
\end{tabular}


Table 1. Cont

\begin{tabular}{|c|c|c|c|c|c|c|c|c|c|c|c|c|c|c|c|}
\hline & \multicolumn{3}{|c|}{ Total } & \multicolumn{3}{|c|}{$<20$ years } & \multicolumn{3}{|c|}{$20<$ years $<50$} & \multicolumn{3}{|c|}{$50<$ years $<70$} & \multicolumn{3}{|c|}{$>70$ years } \\
\hline & $n$ & $\begin{array}{l}\text { Mean } \\
(\mathrm{SE})\end{array}$ & $\begin{array}{l}\text { Median } \\
\text { (Range) }\end{array}$ & $n$ & $\begin{array}{l}\text { Mean } \\
\text { (SE) }\end{array}$ & $\begin{array}{l}\text { Median } \\
\text { (Range) }\end{array}$ & $n$ & $\begin{array}{l}\text { Mean } \\
\text { (SE) }\end{array}$ & $\begin{array}{l}\text { Median } \\
\text { (Range) }\end{array}$ & $n$ & $\begin{array}{l}\text { Mean } \\
\text { (SE) }\end{array}$ & $\begin{array}{l}\text { Median } \\
\text { (Range) }\end{array}$ & $n$ & $\begin{array}{l}\text { Mean } \\
\text { (SE) }\end{array}$ & $\begin{array}{l}\text { Median } \\
\text { (Range) }\end{array}$ \\
\hline \multicolumn{16}{|c|}{ Total protein } \\
\hline Total & 1838 & $\begin{array}{l}83.98 \\
(0.66)\end{array}$ & $\begin{array}{r}80.02 \\
(29.27 \\
332.66)\end{array}$ & 53 & $\begin{array}{l}86.24 \\
(3.14)\end{array}$ & $\begin{array}{r}83.52 \\
(33.65 \\
138.33)\end{array}$ & 963 & $\begin{array}{l}84.27 \\
(0.96)\end{array}$ & $\begin{array}{c}79.31 \\
(29.27 \\
332.66)\end{array}$ & 597 & $\begin{array}{l}83.89 \\
(1.14)\end{array}$ & $\begin{array}{r}80.18 \\
(29.35 \\
303.04)\end{array}$ & 225 & $\begin{array}{l}82.41 \\
(1.63)\end{array}$ & $\begin{array}{c}80.23 \\
(29.35 \\
185.58)\end{array}$ \\
\hline Men & 772 & $\begin{array}{l}85.22 \\
(1.11)\end{array}$ & $\begin{array}{r}79.67 \\
(33.65 \\
332.66)\end{array}$ & 30 & $\begin{array}{l}88.27 \\
(4.03)\end{array}$ & $\begin{array}{r}89.30 \\
(33.65 \\
137.17)\end{array}$ & 384 & $\begin{array}{l}85.01 \\
(1.63)\end{array}$ & $\begin{array}{c}79.21 \\
(43.56 \\
332.66)\end{array}$ & 265 & $\begin{array}{l}85.59 \\
(1.96)\end{array}$ & $\begin{array}{r}79.54 \\
(37.92 \\
303.04)\end{array}$ & 93 & $\begin{array}{l}84.07 \\
(2.56)\end{array}$ & $\begin{array}{c}80.73 \\
(39.51 \\
185.58)\end{array}$ \\
\hline Women & 1066 & $\begin{array}{l}83.07 \\
(0.81)\end{array}$ & $\begin{array}{r}80.23 \\
(29.27 \\
215.96)\end{array}$ & 23 & $\begin{array}{l}83.59 \\
(5.01)\end{array}$ & $\begin{array}{c}78.86 \\
(50.69 \\
138.33)\end{array}$ & 579 & $\begin{array}{l}83.78 \\
(1.17)\end{array}$ & $\begin{array}{c}80.38 \\
(29.27 \\
215.96)\end{array}$ & 332 & $\begin{array}{l}82.53 \\
(1.32)\end{array}$ & $\begin{array}{r}80.24 \\
(29.35 \\
185.93)\end{array}$ & 132 & $\begin{array}{l}81.25 \\
(2.12)\end{array}$ & $\begin{array}{r}79.47 \\
(29.35 \\
156.18) \\
\end{array}$ \\
\hline \multicolumn{16}{|l|}{$\begin{array}{l}\text { Animal } \\
\text { protein }\end{array}$} \\
\hline Total & 1838 & $\begin{array}{l}25.65 \\
(0.44)\end{array}$ & $\begin{array}{c}22.75 \\
(0.00 \\
449.25)\end{array}$ & 53 & $\begin{array}{l}30.68 \\
(1.83)\end{array}$ & $\begin{array}{l}30.97 \\
(6.63 \\
68.99) \\
\end{array}$ & 963 & $\begin{array}{l}26.03 \\
(0.68)\end{array}$ & $\begin{array}{c}23.08 \\
(0.00 \\
449.25)\end{array}$ & 597 & $\begin{array}{l}25.73 \\
(0.74)\end{array}$ & $\begin{array}{c}22.63 \\
(0.00 \\
238.85)\end{array}$ & 225 & $\begin{array}{c}22.67 \\
(0.77) *\end{array}$ & $\begin{array}{l}18.98 \\
(3.05, \\
61.61)\end{array}$ \\
\hline Men & 772 & $\begin{array}{l}26.46 \\
(0.67)\end{array}$ & $\begin{array}{c}23.07 \\
(0.00 \\
238.85)\end{array}$ & 30 & $\begin{array}{l}31.26 \\
(2.85)\end{array}$ & $\begin{array}{l}29.70 \\
(6.63 \\
68.99)\end{array}$ & 384 & $\begin{array}{l}26.88 \\
(0.91)\end{array}$ & $\begin{array}{c}23.55 \\
(0.00 \\
161.09)\end{array}$ & 265 & $\begin{array}{l}26.46 \\
(1.32)\end{array}$ & $\begin{array}{c}23.20 \\
(0.00 \\
238.85)\end{array}$ & 93 & $\begin{array}{l}23.13 \\
(1.09)\end{array}$ & $\begin{array}{l}20.69 \\
(6.23 \\
54.31)\end{array}$ \\
\hline Women & 1066 & $\begin{array}{l}25.07 \\
(0.59)\end{array}$ & $\begin{array}{c}22.38 \\
(0.00 \\
449.25)\end{array}$ & 23 & $\begin{array}{l}29.93 \\
(2.08)\end{array}$ & $\begin{array}{l}31.34 \\
(7.14, \\
47.55)\end{array}$ & 579 & $\begin{array}{l}25.46 \\
(0.95)\end{array}$ & $\begin{array}{c}22.91 \\
(0.00 \\
449.25)\end{array}$ & 332 & $\begin{array}{l}25.15 \\
(0.81)\end{array}$ & $\begin{array}{r}22.19 \\
(5.25 \\
148.42)\end{array}$ & 132 & $\begin{array}{l}22.35 \\
(1.07)\end{array}$ & $\begin{array}{l}18.71 \\
(3.05, \\
61.61)\end{array}$ \\
\hline
\end{tabular}


Table 1. Cont.

\begin{tabular}{|c|c|c|c|c|c|c|c|c|c|c|c|c|c|c|c|}
\hline & \multicolumn{3}{|c|}{ Total } & \multicolumn{3}{|c|}{$<20$ years } & \multicolumn{3}{|c|}{$20<$ years $<50$} & \multicolumn{3}{|c|}{$50<$ years $<70$} & \multicolumn{3}{|c|}{$>70$ years } \\
\hline & $n$ & $\begin{array}{c}\text { Mean } \\
\text { (SE) }\end{array}$ & $\begin{array}{l}\text { Median } \\
\text { (Range) }\end{array}$ & $n$ & $\begin{array}{c}\text { Mean } \\
\text { (SE) }\end{array}$ & $\begin{array}{l}\text { Median } \\
\text { (Range) }\end{array}$ & $n$ & $\begin{array}{c}\text { Mean } \\
\text { (SE) }\end{array}$ & $\begin{array}{l}\text { Median } \\
\text { (Range) }\end{array}$ & $n$ & $\begin{array}{l}\text { Mean } \\
\text { (SE) }\end{array}$ & $\begin{array}{l}\text { Median } \\
\text { (Range) }\end{array}$ & $n$ & $\begin{array}{l}\text { Mean } \\
\text { (SE) }\end{array}$ & $\begin{array}{l}\text { Median } \\
\text { (Range) }\end{array}$ \\
\hline \multicolumn{16}{|c|}{ Dairy protein } \\
\hline Total & 1838 & $\begin{array}{l}14.01 \\
(0.21)\end{array}$ & $\begin{array}{l}12.24 \\
(0.00 \\
67.63) \\
\end{array}$ & 53 & $\begin{array}{l}14.84 \\
(1.02)\end{array}$ & $\begin{array}{l}12.69 \\
(0.00 \\
28.48)\end{array}$ & 963 & $\begin{array}{l}14.19 \\
(0.30)\end{array}$ & $\begin{array}{l}11.78 \\
(0.00 \\
67.63) \\
\end{array}$ & 597 & $\begin{array}{l}13.75 \\
(0.34)\end{array}$ & $\begin{array}{l}12.85 \\
(0.00 \\
52.81) \\
\end{array}$ & 225 & $\begin{array}{l}13.69 \\
(0.53)\end{array}$ & $\begin{array}{l}13.09 \\
(0.00 \\
50.39) \\
\end{array}$ \\
\hline Men & 772 & $\begin{array}{l}13.59 \\
(0.32)\end{array}$ & $\begin{array}{l}11.60 \\
(0.00 \\
67.63)\end{array}$ & 30 & $\begin{array}{l}13.69 \\
(1.55)\end{array}$ & $\begin{array}{l}11.91 \\
(0.00 \\
28.48)\end{array}$ & 384 & $\begin{array}{l}13.70 \\
(0.52)\end{array}$ & $\begin{array}{l}10.76 \\
(0.00 \\
67.63)\end{array}$ & 265 & $\begin{array}{l}13.17 \\
(0.48)\end{array}$ & $\begin{array}{l}13.08 \\
(0.00 \\
44.02)\end{array}$ & 93 & $\begin{array}{l}14.28 \\
(0.78)\end{array}$ & $\begin{array}{l}15.25 \\
(0.00 \\
42.42)\end{array}$ \\
\hline Women & 1066 & $\begin{array}{l}14.31 \\
(0.27)\end{array}$ & $\begin{array}{l}12.71 \\
(0.00 \\
54.33)\end{array}$ & 23 & $\begin{array}{l}16.35 \\
(1.17)\end{array}$ & $\begin{array}{l}15.53 \\
(8.52 \\
27.50)\end{array}$ & 579 & $\begin{array}{l}14.52 \\
(0.37)\end{array}$ & $\begin{array}{l}12.77 \\
(0.00 \\
54.33)\end{array}$ & 332 & $\begin{array}{l}14.21 \\
(0.47)\end{array}$ & $\begin{array}{l}12.39 \\
(0.00 \\
52.81)\end{array}$ & 132 & $\begin{array}{l}13.27 \\
(0.72)\end{array}$ & $\begin{array}{l}12.04 \\
(0.00 \\
50.39)\end{array}$ \\
\hline \multicolumn{16}{|c|}{ Plant protein } \\
\hline Total & 1838 & $\begin{array}{c}44.71 \\
(0.41,)\end{array}$ & $\begin{array}{c}41.90 \\
(6.90 \\
178.86)\end{array}$ & 53 & $\begin{array}{l}44.13 \\
(1.89)\end{array}$ & $\begin{array}{r}43.91 \\
(6.90 \\
86.56) \\
\end{array}$ & 963 & $\begin{array}{l}45.11 \\
(0.61)\end{array}$ & $\begin{array}{r}41.92 \\
(13.67 \\
178.86)\end{array}$ & 597 & $\begin{array}{l}44.69 \\
(0.70)\end{array}$ & $\begin{array}{r}41.88 \\
(11.96 \\
140.10)\end{array}$ & 225 & $\begin{array}{l}43.16 \\
(0.99)\end{array}$ & $\begin{array}{c}40.57 \\
(15.11 \\
83.01) \\
\end{array}$ \\
\hline Men & 772 & $\begin{array}{l}45.35 \\
(0.67)\end{array}$ & $\begin{array}{c}42.14 \\
(6.90 \\
178.86)\end{array}$ & 30 & $\begin{array}{l}44.75 \\
(2.53)\end{array}$ & $\begin{array}{l}45.04 \\
(6.90 \\
70.78) \\
\end{array}$ & 384 & $\begin{array}{l}45.93 \\
(1.02)\end{array}$ & $\begin{array}{r}42.46 \\
(13.67 \\
178.86)\end{array}$ & 265 & $\begin{array}{l}45.19 \\
(1.11)\end{array}$ & $\begin{array}{r}41.79 \\
(16.35 \\
140.10)\end{array}$ & 93 & $\begin{array}{l}43.64 \\
(1.55)\end{array}$ & $\begin{array}{l}40.55 \\
(17.71, \\
82.61)\end{array}$ \\
\hline Women & 1066 & $\begin{array}{l}44.24 \\
(0.52)\end{array}$ & $\begin{array}{c}41.80 \\
(11.96, \\
117.03)\end{array}$ & 23 & $\begin{array}{l}43.32 \\
(2.91)\end{array}$ & $\begin{array}{l}40.89 \\
(25.91, \\
86.56)\end{array}$ & 579 & $\begin{array}{l}44.57 \\
(0.74)\end{array}$ & $\begin{array}{r}41.69 \\
(16.36 \\
117.03)\end{array}$ & 332 & $\begin{array}{l}44.29 \\
(0.89)\end{array}$ & $\begin{array}{r}42.31 \\
(11.96 \\
100.77)\end{array}$ & 132 & $\begin{array}{l}42.82 \\
(1.30)\end{array}$ & $\begin{array}{l}40.62 \\
(15.11, \\
83.01)\end{array}$ \\
\hline
\end{tabular}


Table 1. Cont.

\begin{tabular}{|c|c|c|c|c|c|c|c|c|c|c|c|c|c|c|c|}
\hline & \multicolumn{3}{|c|}{ Total } & \multicolumn{3}{|c|}{$<20$ years } & \multicolumn{3}{|c|}{$20<$ years $<50$} & \multicolumn{3}{|c|}{$50<$ years $<70$} & \multicolumn{3}{|c|}{$>70$ years } \\
\hline & $n$ & $\begin{array}{l}\text { Mean } \\
\text { (SE) }\end{array}$ & $\begin{array}{l}\text { Median } \\
\text { (Range) }\end{array}$ & $n$ & $\begin{array}{l}\text { Mean } \\
\text { (SE) }\end{array}$ & $\begin{array}{l}\text { Median } \\
\text { (Range) }\end{array}$ & $n$ & $\begin{array}{l}\text { Mean } \\
\text { (SE) }\end{array}$ & $\begin{array}{l}\text { Median } \\
\text { (Range) }\end{array}$ & $n$ & $\begin{array}{l}\text { Mean } \\
\text { (SE) }\end{array}$ & $\begin{array}{l}\text { Median } \\
\text { (Range) }\end{array}$ & $n$ & $\begin{array}{l}\text { Mean } \\
\text { (SE) }\end{array}$ & $\begin{array}{l}\text { Median } \\
\text { (Range) }\end{array}$ \\
\hline \multicolumn{16}{|c|}{$\begin{array}{c}\text { Total } \\
\text { carbohydrates }\end{array}$} \\
\hline Total & 1838 & $\begin{array}{c}296.02 \\
(2.56)\end{array}$ & $\begin{array}{c}274.18 \\
(100.18 \\
897.76)\end{array}$ & 53 & $\begin{array}{l}289.04 \\
(13.47)\end{array}$ & $\begin{array}{c}275.29 \\
(119.11 \\
590.50)\end{array}$ & 963 & $\begin{array}{l}296.17 \\
(3.69)\end{array}$ & $\begin{array}{c}271.23 \\
(109.87 \\
897.76)\end{array}$ & 597 & $\begin{array}{c}300.52 \\
(4.44)\end{array}$ & $\begin{array}{c}278.50 \\
(100.18 \\
673.97)\end{array}$ & 225 & $\begin{array}{c}285.14 \\
(6.31)\end{array}$ & $\begin{array}{l}268.17 \\
(114.52, \\
560.82)\end{array}$ \\
\hline Men & 772 & $\begin{array}{c}300.69 \\
(4.13)\end{array}$ & $\begin{array}{c}278.62 \\
(109.87 \\
897.76)\end{array}$ & 30 & $\begin{array}{l}290.46 \\
(17.43)\end{array}$ & $\begin{array}{c}275.52 \\
(119.11 \\
482.17)\end{array}$ & 384 & $\begin{array}{c}302.36 \\
(6.33)\end{array}$ & $\begin{array}{c}276.32 \\
(109.87 \\
897.76)\end{array}$ & 265 & $\begin{array}{c}303.96 \\
(6.78)\end{array}$ & $\begin{array}{c}286.63 \\
(126.94 \\
673.97)\end{array}$ & 93 & $\begin{array}{c}287.83 \\
(9.23)\end{array}$ & $\begin{array}{c}269.57 \\
(132.74, \\
504.86)\end{array}$ \\
\hline Women & 1066 & $\begin{array}{l}292.64 \\
(3.25)\end{array}$ & $\begin{array}{c}270.39 \\
(100.18 \\
670.72)\end{array}$ & 23 & $\begin{array}{l}287.19 \\
(21.57)\end{array}$ & $\begin{array}{c}270.44 \\
(137.86 \\
590.50)\end{array}$ & 579 & $\begin{array}{c}292.06 \\
(4.47)\end{array}$ & $\begin{array}{c}270.50 \\
(112.30 \\
670.72)\end{array}$ & 332 & $\begin{array}{l}297.77 \\
(5.86)\end{array}$ & $\begin{array}{c}272.22 \\
(100.18 \\
608.56)\end{array}$ & 132 & $\begin{array}{l}283.24 \\
(8.60)\end{array}$ & $\begin{array}{l}265.18 \\
(114.52, \\
560.82)\end{array}$ \\
\hline \multicolumn{16}{|l|}{ Fiber } \\
\hline Total & 1838 & $\begin{array}{l}31.69 \\
(0.33)\end{array}$ & $\begin{array}{c}29.30 \\
(2.81 \\
150.50)\end{array}$ & 53 & $\begin{array}{l}29.77 \\
(1.55)\end{array}$ & $\begin{array}{l}27.98 \\
(2.81 \\
57.65)\end{array}$ & 963 & $\begin{array}{l}31.81 \\
(0.50)\end{array}$ & $\begin{array}{c}29.11 \\
(6.63, \\
150.50)\end{array}$ & 597 & $\begin{array}{l}32.09 \\
(0.54)\end{array}$ & $\begin{array}{c}30.43 \\
(5.46, \\
100.03)\end{array}$ & 225 & $\begin{array}{l}30.53 \\
(0.79)\end{array}$ & $\begin{array}{l}29.26 \\
(8.31 \\
81.77)\end{array}$ \\
\hline Men & 772 & $\begin{array}{l}32.25 \\
(0.54)\end{array}$ & $\begin{array}{c}29.52 \\
(2.81 \\
150.50)\end{array}$ & 30 & $\begin{array}{l}30.87 \\
(2.18)\end{array}$ & $\begin{array}{l}28.23 \\
(2.81 \\
57.65)\end{array}$ & 384 & $\begin{array}{l}32.23 \\
(0.86)\end{array}$ & $\begin{array}{c}28.34 \\
(9.48 \\
150.50)\end{array}$ & 265 & $\begin{array}{l}32.65 \\
(0.86)\end{array}$ & $\begin{array}{c}30.39 \\
(8.83 \\
100.03)\end{array}$ & 93 & $\begin{array}{l}31.65 \\
(1.14)\end{array}$ & $\begin{array}{l}30.36 \\
(11.01, \\
57.97)\end{array}$ \\
\hline Women & 1066 & $\begin{array}{l}31.28 \\
(0.42)\end{array}$ & $\begin{array}{l}29.23 \\
(5.46 \\
85.11)\end{array}$ & 23 & $\begin{array}{l}28.34 \\
(2.17)\end{array}$ & $\begin{array}{l}26.42 \\
(12.77 \\
56.68)\end{array}$ & 579 & $\begin{array}{l}31.54 \\
(0.60)\end{array}$ & $\begin{array}{c}29.28 \\
(6.63, \\
85.11)\end{array}$ & 332 & $\begin{array}{l}31.63 \\
(0.68)\end{array}$ & $\begin{array}{l}30.46 \\
(5.46 \\
78.36)\end{array}$ & 132 & $\begin{array}{l}29.73 \\
(1.08)\end{array}$ & $\begin{array}{l}27.75 \\
(8.31 \\
81.77)\end{array}$ \\
\hline
\end{tabular}


Table 2. Total, sex, and age group-specific consumption of animal food groups in the study participants of the MEAL study $(n=1838) .{ }^{*}$ denotes $p<0.05, * *$ denotes $p<0.001$.

\begin{tabular}{|c|c|c|c|c|c|c|c|c|c|c|c|c|c|c|c|}
\hline & \multicolumn{3}{|c|}{ Total } & \multicolumn{3}{|c|}{$<20$ years } & \multicolumn{3}{|c|}{$20<$ years $<50$} & \multicolumn{3}{|c|}{$50<$ years $<70$} & \multicolumn{3}{|c|}{$>70$ years } \\
\hline & $n$ & $\begin{array}{l}\text { Mean } \\
\text { (SE) }\end{array}$ & $\begin{array}{l}\text { Median } \\
\text { (Range) }\end{array}$ & $n$ & $\begin{array}{l}\text { Mean } \\
\text { (SE) }\end{array}$ & $\begin{array}{l}\text { Median } \\
\text { (Range) }\end{array}$ & $n$ & $\begin{array}{l}\text { Mean } \\
\text { (SE) }\end{array}$ & $\begin{array}{l}\text { Median } \\
\text { (Range) }\end{array}$ & $n$ & $\begin{array}{l}\text { Mean } \\
\text { (SE) }\end{array}$ & $\begin{array}{l}\text { Median } \\
\text { (Range) }\end{array}$ & $n$ & $\begin{array}{l}\text { Mean } \\
\text { (SE) }\end{array}$ & $\begin{array}{l}\text { Median } \\
\text { (Range) }\end{array}$ \\
\hline \multicolumn{16}{|c|}{$\begin{array}{l}\text { Total processed } \\
\text { meats }\end{array}$} \\
\hline Total & 1838 & $\begin{array}{c}15.99 \\
(0.43)^{* *}\end{array}$ & $\begin{array}{c}11.50 \\
(0.00 \\
168.00)\end{array}$ & 53 & $\begin{array}{l}16.82 \\
(1.92)\end{array}$ & $\begin{array}{l}17.05 \\
(0.00 \\
53.00)\end{array}$ & 963 & $\begin{array}{l}17.57 \\
(0.58)\end{array}$ & $\begin{array}{c}11.50 \\
(0.00 \\
129.50)\end{array}$ & 597 & $\begin{array}{l}14.54 \\
(0.82)\end{array}$ & $\begin{array}{c}7.00 \\
(0.00 \\
168.00)\end{array}$ & 225 & $\begin{array}{l}12.85 \\
(0.99)\end{array}$ & $\begin{array}{c}7.00 \\
(0.00 \\
157.00)\end{array}$ \\
\hline Men & 772 & $\begin{array}{l}18.00 \\
(0.75)\end{array}$ & $\begin{array}{c}11.50 \\
(0.00 \\
168.00)\end{array}$ & 30 & $\begin{array}{l}18.68 \\
(2.67)\end{array}$ & $\begin{array}{l}18.00 \\
(0.00 \\
53.00) \\
\end{array}$ & 384 & $\begin{array}{l}19.12 \\
(1.02)\end{array}$ & $\begin{array}{c}11.50 \\
(0.00 \\
129.50)\end{array}$ & 265 & $\begin{array}{l}17.63 \\
(1.49)\end{array}$ & $\begin{array}{c}11.50 \\
(0.00 \\
168.00)\end{array}$ & 93 & $\begin{array}{l}14.24 \\
(1.36)\end{array}$ & $\begin{array}{c}7.85 \\
(0.00 \\
50.00) \\
\end{array}$ \\
\hline Women & 1066 & $\begin{array}{l}14.52 \\
(0.49)\end{array}$ & $\begin{array}{c}7.42 \\
(0.00 \\
157.00) \\
\end{array}$ & 23 & $\begin{array}{l}14.40 \\
(2.73)\end{array}$ & $\begin{array}{c}7.00 \\
(1.50 \\
53.00) \\
\end{array}$ & 579 & $\begin{array}{l}16.54 \\
(0.68)\end{array}$ & $\begin{array}{l}11.50 \\
(0.00 \\
99.35) \\
\end{array}$ & 332 & $\begin{array}{l}12.08 \\
(0.84)\end{array}$ & $\begin{array}{c}7.00 \\
(0.00 \\
129.50)\end{array}$ & 132 & $\begin{array}{l}11.87 \\
(1.39)\end{array}$ & $\begin{array}{c}7.00 \\
(0.00, \\
157.00) \\
\end{array}$ \\
\hline \multicolumn{16}{|c|}{$\begin{array}{l}\text { Unprocessed } \\
\text { meats }\end{array}$} \\
\hline Total & 1838 & $\begin{array}{l}33.78 \\
(0.59)\end{array}$ & $\begin{array}{c}28.00 \\
(0.00 \\
286.00)\end{array}$ & 53 & $\begin{array}{l}38.01 \\
(4.06)\end{array}$ & $\begin{array}{c}28.00 \\
(0.00 \\
114.00)\end{array}$ & 963 & $\begin{array}{l}33.58 \\
(0.76)\end{array}$ & $\begin{array}{c}28.00 \\
(0.00 \\
136.00)\end{array}$ & 597 & $\begin{array}{l}33.78 \\
(1.10)\end{array}$ & $\begin{array}{c}28.00 \\
(0.00 \\
286.00)\end{array}$ & 225 & $\begin{array}{l}33.67 \\
(1.85)\end{array}$ & $\begin{array}{c}28.00 \\
(0.00 \\
164.00)\end{array}$ \\
\hline Men & 772 & $\begin{array}{l}34.65 \\
(0.65)\end{array}$ & $\begin{array}{c}28.00 \\
(0.00 \\
286.00)\end{array}$ & 30 & $\begin{array}{l}35.06 \\
(5.18)\end{array}$ & $\begin{array}{c}28.00 \\
(0.00 \\
100.00)\end{array}$ & 384 & $\begin{array}{l}33.58 \\
(1.19)\end{array}$ & $\begin{array}{c}28.00 \\
(0.00 \\
128.00)\end{array}$ & 265 & $\begin{array}{l}36.23 \\
(1.80)\end{array}$ & $\begin{array}{c}28.00 \\
(0.00 \\
286.00)\end{array}$ & 93 & $\begin{array}{l}34.40 \\
(2.96)\end{array}$ & $\begin{array}{c}28.00 \\
(3.00 \\
164.00) \\
\end{array}$ \\
\hline Women & 1066 & $\begin{array}{l}33.16 \\
(0.75)\end{array}$ & $\begin{array}{c}28.00 \\
(0.00 \\
164.00)\end{array}$ & 23 & $\begin{array}{l}41.86 \\
(6.51)\end{array}$ & $\begin{array}{c}28.00 \\
(0.00 \\
114.00)\end{array}$ & 579 & $\begin{array}{l}33.58 \\
(0.99)\end{array}$ & $\begin{array}{c}28.00 \\
(0.00 \\
136.00)\end{array}$ & 332 & $\begin{array}{l}31.82 \\
(1.34)\end{array}$ & $\begin{array}{c}28.00 \\
(0.00 \\
136.00)\end{array}$ & 132 & $\begin{array}{l}33.16 \\
(2.36)\end{array}$ & $\begin{array}{c}28.00 \\
(0.00 \\
164.00)\end{array}$ \\
\hline
\end{tabular}


Table 2. Cont

\begin{tabular}{|c|c|c|c|c|c|c|c|c|c|c|c|c|c|c|c|}
\hline & \multicolumn{3}{|c|}{ Total } & \multicolumn{3}{|c|}{$<20$ years } & \multicolumn{3}{|c|}{$20<$ years $<50$} & \multicolumn{3}{|c|}{$50<$ years $<70$} & \multicolumn{3}{|c|}{ >70 years } \\
\hline & $n$ & $\begin{array}{l}\text { Mean } \\
\text { (SE) }\end{array}$ & $\begin{array}{l}\text { Median } \\
\text { (Range) }\end{array}$ & $n$ & $\begin{array}{l}\text { Mean } \\
\text { (SE) }\end{array}$ & $\begin{array}{l}\text { Median } \\
\text { (Range) }\end{array}$ & $n$ & $\begin{array}{l}\text { Mean } \\
\text { (SE) }\end{array}$ & $\begin{array}{l}\text { Median } \\
\text { (Range) }\end{array}$ & $n$ & $\begin{array}{c}\text { Mean } \\
\text { (SE) }\end{array}$ & $\begin{array}{l}\text { Median } \\
\text { (Range) }\end{array}$ & $n$ & $\begin{array}{l}\text { Mean } \\
\text { (SE) }\end{array}$ & $\begin{array}{l}\text { Median } \\
\text { (Range) }\end{array}$ \\
\hline \multicolumn{16}{|c|}{ Total seafood } \\
\hline Total & 1838 & $\begin{array}{l}60.81 \\
(1.28)\end{array}$ & $\begin{array}{c}47.40 \\
(0.00 \\
784.70)\end{array}$ & 53 & $\begin{array}{l}60.35 \\
(5.30)\end{array}$ & $\begin{array}{c}54.70 \\
(0.00 \\
145.00)\end{array}$ & 963 & $\begin{array}{l}59.17 \\
(1.85)\end{array}$ & $\begin{array}{c}45.00 \\
(0.00 \\
784.70)\end{array}$ & 597 & $\begin{array}{l}63.71 \\
(2.19)\end{array}$ & $\begin{array}{c}50.40 \\
(0.00 \\
442.00)\end{array}$ & 225 & $\begin{array}{l}60.26 \\
(3.45)\end{array}$ & $\begin{array}{c}48.10 \\
(0.00 \\
448.00)\end{array}$ \\
\hline Men & 772 & $\begin{array}{l}61.07 \\
(2.18)\end{array}$ & $\begin{array}{c}46.80 \\
(0.00 \\
784.70)\end{array}$ & 30 & $\begin{array}{l}56.56 \\
(7.47)\end{array}$ & $\begin{array}{c}46.50 \\
(0.00 \\
142.00)\end{array}$ & 384 & $\begin{array}{l}58.41 \\
(3.28)\end{array}$ & $\begin{array}{c}43.30 \\
(0.00 \\
784.70)\end{array}$ & 265 & $\begin{array}{l}65.84 \\
(3.55)\end{array}$ & $\begin{array}{c}54.10 \\
(3.00 \\
442.00)\end{array}$ & 93 & $\begin{array}{l}59.91 \\
(6.03)\end{array}$ & $\begin{array}{c}45.00 \\
(6.00, \\
448.00)\end{array}$ \\
\hline Women & 1066 & $\begin{array}{l}60.62 \\
(1.55)\end{array}$ & $\begin{array}{c}48.00 \\
(0.00 \\
408.00)\end{array}$ & 23 & $\begin{array}{l}65.29 \\
(7.40)\end{array}$ & $\begin{array}{r}58.40 \\
(12.70 \\
145.00)\end{array}$ & 579 & $\begin{array}{l}59.68 \\
(2.18)\end{array}$ & $\begin{array}{c}47.40 \\
(0.00 \\
408.00)\end{array}$ & 332 & $\begin{array}{l}62.01 \\
(2.72)\end{array}$ & $\begin{array}{c}47.70 \\
(0.00 \\
373.70)\end{array}$ & 132 & $\begin{array}{l}60.50 \\
(4.09)\end{array}$ & $\begin{array}{c}50.45 \\
(0.00 \\
250.00)\end{array}$ \\
\hline \multicolumn{16}{|l|}{ Eggs } \\
\hline Total & 1838 & $\begin{array}{c}2.38 \\
(0.11) *\end{array}$ & $\begin{array}{c}0.77 \\
(0.00 \\
24.75)\end{array}$ & 53 & $\begin{array}{c}1.84 \\
(0.38)\end{array}$ & $\begin{array}{c}0.77 \\
(0.00 \\
13.75)\end{array}$ & 963 & $\begin{array}{c}1.92 \\
(0.12)\end{array}$ & $\begin{array}{c}0.77 \\
(0.00 \\
24,75)\end{array}$ & 597 & $\begin{array}{c}2.80 \\
(0.22)\end{array}$ & $\begin{array}{c}0.77 \\
(0.00 \\
24.75)\end{array}$ & 225 & $\begin{array}{c}3.30 \\
(0.38)^{* *}\end{array}$ & $\begin{array}{c}0.77 \\
(0.00 \\
24.75)\end{array}$ \\
\hline Men & 772 & $\begin{array}{c}2.04 \\
(0.14)\end{array}$ & $\begin{array}{c}0.77 \\
(0.00 \\
24.75)\end{array}$ & 30 & $\begin{array}{c}1.72 \\
(0.48)\end{array}$ & $\begin{array}{c}0.77 \\
(0.00 \\
13.75)\end{array}$ & 384 & $\begin{array}{c}1.42 \\
(0.11)\end{array}$ & $\begin{array}{c}0.77 \\
(0.00 \\
24.75)\end{array}$ & 265 & $\begin{array}{c}2.27 \\
(0.27)\end{array}$ & $\begin{array}{c}0.77 \\
(0.00 \\
24.75)\end{array}$ & 93 & $\begin{array}{c}4.08 \\
(0.67)\end{array}$ & $\begin{array}{c}1.98 \\
(0.00 \\
24.75)\end{array}$ \\
\hline Women & 1066 & $\begin{array}{c}2.62 \\
(0.16)\end{array}$ & $\begin{array}{c}0.77 \\
(0.00 \\
24.75)\end{array}$ & 23 & $\begin{array}{c}2.00 \\
(0.61)\end{array}$ & $\begin{array}{c}0.77 \\
(0.16 \\
13.75)\end{array}$ & 579 & $\begin{array}{c}2.26 \\
(0.19)\end{array}$ & $\begin{array}{c}0.77 \\
(0.00 \\
24.75)\end{array}$ & 332 & $\begin{array}{c}3.23 \\
(0.32)\end{array}$ & $\begin{array}{c}0.77 \\
(0.00 \\
24.75)\end{array}$ & 132 & $\begin{array}{c}2.75 \\
(0.44)\end{array}$ & $\begin{array}{c}0.77 \\
(0.00, \\
24.75)\end{array}$ \\
\hline \multicolumn{16}{|l|}{ Cheese } \\
\hline Total & 1838 & $\begin{array}{l}53.45 \\
(0.80)\end{array}$ & $\begin{array}{c}46.70 \\
(0.00 \\
328.01)\end{array}$ & 53 & $\begin{array}{l}56.29 \\
(4.27)\end{array}$ & $\begin{array}{c}50.20 \\
(15.51 \\
147.47)\end{array}$ & 963 & $\begin{array}{l}53.68 \\
(1.13)\end{array}$ & $\begin{array}{c}46.82 \\
(0.00 \\
310.01)\end{array}$ & 597 & $\begin{array}{l}53.49 \\
(1.43)\end{array}$ & $\begin{array}{c}46.08 \\
(0.00 \\
328.01)\end{array}$ & 225 & $\begin{array}{l}51.74 \\
(2.02)\end{array}$ & $\begin{array}{c}46.33 \\
(0.00 \\
231.88)\end{array}$ \\
\hline
\end{tabular}


Table 2. Cont

\begin{tabular}{|c|c|c|c|c|c|c|c|c|c|c|c|c|c|c|c|}
\hline & \multicolumn{3}{|c|}{ Total } & \multicolumn{3}{|c|}{$<20$ years } & \multicolumn{3}{|c|}{$20<$ years $<50$} & \multicolumn{3}{|c|}{$50<$ years $<70$} & \multicolumn{3}{|c|}{$>70$ years } \\
\hline & $n$ & $\begin{array}{l}\text { Mean } \\
\text { (SE) }\end{array}$ & $\begin{array}{l}\text { Median } \\
\text { (Range) }\end{array}$ & $n$ & $\begin{array}{l}\text { Mean } \\
\text { (SE) }\end{array}$ & $\begin{array}{l}\text { Median } \\
\text { (Range) }\end{array}$ & $n$ & $\begin{array}{l}\text { Mean } \\
\text { (SE) }\end{array}$ & $\begin{array}{l}\text { Median } \\
\text { (Range) }\end{array}$ & $n$ & $\begin{array}{l}\text { Mean } \\
\text { (SE) }\end{array}$ & $\begin{array}{l}\text { Median } \\
\text { (Range) }\end{array}$ & $n$ & $\begin{array}{l}\text { Mean } \\
\text { (SE) }\end{array}$ & $\begin{array}{l}\text { Median } \\
\text { (Range) }\end{array}$ \\
\hline Men & 772 & $\begin{array}{l}55.16 \\
(1.30)\end{array}$ & $\begin{array}{c}47.53 \\
(0.00 \\
328.01)\end{array}$ & 30 & $\begin{array}{l}64.43 \\
(5.02)\end{array}$ & $\begin{array}{r}52.67 \\
(26.48, \\
147.47)\end{array}$ & 384 & $\begin{array}{l}53.49 \\
(1.86)\end{array}$ & $\begin{array}{c}44.85 \\
(1.50 \\
310.01)\end{array}$ & 265 & $\begin{array}{l}56.61 \\
(2.35)\end{array}$ & $\begin{array}{c}48.63 \\
(0.00 \\
328.01)\end{array}$ & 93 & $\begin{array}{l}54.98 \\
(3.13)\end{array}$ & $\begin{array}{c}52.08 \\
(0.00, \\
123.20)\end{array}$ \\
\hline Women & 1066 & $\begin{array}{l}52.22 \\
(1.01)\end{array}$ & $\begin{array}{c}45.82 \\
(0.00 \\
296.01)\end{array}$ & 23 & $\begin{array}{l}45.69 \\
(6.84)\end{array}$ & $\begin{array}{c}31.43 \\
(15.51 \\
138.88)\end{array}$ & 579 & $\begin{array}{l}53.80 \\
(1.42)\end{array}$ & $\begin{array}{c}47.50 \\
(0.00 \\
296.01)\end{array}$ & 332 & $\begin{array}{l}51.00 \\
(1.74)\end{array}$ & $\begin{array}{c}43.82 \\
(0.00 \\
213.71)\end{array}$ & 132 & $\begin{array}{l}49.46 \\
(2.64)\end{array}$ & $\begin{array}{c}45.72 \\
(0.00, \\
231.88)\end{array}$ \\
\hline \multicolumn{16}{|l|}{ Yoghurt } \\
\hline Total & 1838 & $\begin{array}{l}28.79 \\
(1.07)\end{array}$ & $\begin{array}{c}8.38 \\
(0.00 \\
312.50)\end{array}$ & 53 & $\begin{array}{l}37.23 \\
(9.20)\end{array}$ & $\begin{array}{c}8.38 \\
(0.00 \\
312.50)\end{array}$ & 963 & $\begin{array}{l}26.83 \\
(1.36)\end{array}$ & $\begin{array}{c}8.38 \\
(0.00 \\
312.50)\end{array}$ & 597 & $\begin{array}{l}29.71 \\
(1.88)\end{array}$ & $\begin{array}{c}8.38 \\
(0.00 \\
312.50)\end{array}$ & 225 & $\begin{array}{l}32.77 \\
(3.57)\end{array}$ & $\begin{array}{c}8.38 \\
(0.00 \\
312.50)\end{array}$ \\
\hline Men & 772 & $\begin{array}{l}28.27 \\
(1.66)\end{array}$ & $\begin{array}{c}8.38 \\
(0.00 \\
312.50)\end{array}$ & 30 & $\begin{array}{c}50.05 \\
(14.85)\end{array}$ & $\begin{array}{c}17.50 \\
(0.00 \\
312.50)\end{array}$ & 384 & $\begin{array}{l}26.31 \\
(2.09)\end{array}$ & $\begin{array}{c}8.38 \\
(0.00 \\
312.50)\end{array}$ & 265 & $\begin{array}{l}27.70 \\
(2.79)\end{array}$ & $\begin{array}{c}8.38 \\
(0.00 \\
312.50)\end{array}$ & 93 & $\begin{array}{l}30.96 \\
(5.28)\end{array}$ & $\begin{array}{c}8.38 \\
(0.00 \\
312.50)\end{array}$ \\
\hline Women & 1066 & $\begin{array}{l}29.17 \\
(1.40)\end{array}$ & $\begin{array}{c}8.38 \\
(0.00 \\
312.50)\end{array}$ & 23 & $\begin{array}{l}20.51 \\
(7.72)\end{array}$ & $\begin{array}{c}0.00 \\
(0.00 \\
125.00)\end{array}$ & 579 & $\begin{array}{l}27.18 \\
(1.79)\end{array}$ & $\begin{array}{c}8.38 \\
(0.00 \\
312.50)\end{array}$ & 332 & $\begin{array}{l}31.32 \\
(2.55)\end{array}$ & $\begin{array}{c}8.38 \\
(0.00 \\
312.50)\end{array}$ & 132 & $\begin{array}{l}34.04 \\
(4.83)\end{array}$ & $\begin{array}{c}8.38 \\
(0.00 \\
312.50)\end{array}$ \\
\hline \multicolumn{16}{|c|}{$\begin{array}{l}\text { Reduced fat } \\
\text { milk }\end{array}$} \\
\hline Total & 1838 & $\begin{array}{l}124.71 \\
(3.68)\end{array}$ & $\begin{array}{c}90.00 \\
(0.00 \\
1125.00)\end{array}$ & 53 & $\begin{array}{c}129.83 \\
(19.42)\end{array}$ & $\begin{array}{c}90.00 \\
(0.00 \\
625.00)\end{array}$ & 963 & $\begin{array}{l}127.22 \\
(5.16)\end{array}$ & $\begin{array}{c}90.00 \\
(0.00 \\
1125,00)\end{array}$ & 597 & $\begin{array}{l}118.01 \\
(6.28)\end{array}$ & $\begin{array}{c}90.00 \\
(0.00 \\
1125.00)\end{array}$ & 225 & $\begin{array}{l}130.56 \\
(10.82)\end{array}$ & $\begin{array}{c}90.00 \\
(0.00 \\
625.00)\end{array}$ \\
\hline Men & 772 & $\begin{array}{l}121.70 \\
(5.48)\end{array}$ & $\begin{array}{c}90.00 \\
(0.00 \\
1125.00)\end{array}$ & 30 & $\begin{array}{l}125.20 \\
(20.49)\end{array}$ & $\begin{array}{c}90.00 \\
(0.00 \\
250.00)\end{array}$ & 384 & $\begin{array}{l}130.70 \\
(8.31)\end{array}$ & $\begin{array}{c}90.00 \\
(0.00 \\
1125.00)\end{array}$ & 265 & $\begin{array}{c}107.36 \\
(8.60)\end{array}$ & $\begin{array}{c}35.00 \\
(0.00 \\
625.00)\end{array}$ & 93 & $\begin{array}{l}124.31 \\
(15.55)\end{array}$ & $\begin{array}{c}90.00 \\
(0.00 \\
625.00)\end{array}$ \\
\hline Women & 1066 & $\begin{array}{l}126.89 \\
(4.95)\end{array}$ & $\begin{array}{c}90.00 \\
(0.00 \\
1125.00)\end{array}$ & 23 & $\begin{array}{l}135.87 \\
(36.48)\end{array}$ & $\begin{array}{c}90.00 \\
(0.00 \\
625.00)\end{array}$ & 579 & $\begin{array}{l}124.91 \\
(6.59)\end{array}$ & $\begin{array}{c}90.00 \\
(0.00 \\
625.00)\end{array}$ & 332 & $\begin{array}{c}126.52 \\
(8.94)\end{array}$ & $\begin{array}{c}90.00 \\
(0.00 \\
1125.00)\end{array}$ & 132 & $\begin{array}{l}134.96 \\
(14.88)\end{array}$ & $\begin{array}{c}90.00 \\
(0.00 \\
625.00)\end{array}$ \\
\hline
\end{tabular}


Table 3. Total, sex, and age group-specific consumption of plant food groups in the study participants of the MEAL study $(n=1838) .{ }^{*}$ denotes $p<0.05$.

\begin{tabular}{|c|c|c|c|c|c|c|c|c|c|c|c|c|c|c|c|}
\hline & \multicolumn{3}{|c|}{ Total } & \multicolumn{3}{|c|}{$<20$ years } & \multicolumn{3}{|c|}{$20<$ years $<50$} & \multicolumn{3}{|c|}{$50<$ years $<70$} & \multicolumn{3}{|c|}{$>70$ years } \\
\hline & $n$ & $\begin{array}{l}\text { Mean } \\
\text { (SE) }\end{array}$ & $\begin{array}{l}\text { Median } \\
\text { (Range) }\end{array}$ & $n$ & $\begin{array}{l}\text { Mean } \\
\text { (SE) }\end{array}$ & $\begin{array}{l}\text { Median } \\
\text { (Range) }\end{array}$ & $n$ & $\begin{array}{l}\text { Mean } \\
\text { (SE) }\end{array}$ & $\begin{array}{l}\text { Median } \\
\text { (Range) }\end{array}$ & $n$ & $\begin{array}{l}\text { Mean } \\
\text { (SE) }\end{array}$ & $\begin{array}{l}\text { Median } \\
\text { (Range) }\end{array}$ & $n$ & $\begin{array}{l}\text { Mean } \\
\text { (SE) }\end{array}$ & $\begin{array}{l}\text { Median } \\
\text { (Range) }\end{array}$ \\
\hline \multicolumn{16}{|l|}{ Fruits } \\
\hline Total & 1838 & $\begin{array}{c}395.92 \\
(7.43)\end{array}$ & $\begin{array}{c}295.13 \\
(0.00 \\
2801.47)\end{array}$ & 53 & $\begin{array}{l}335.51 \\
(28.08)\end{array}$ & $\begin{array}{c}303.58 \\
(0.00 \\
951.57)\end{array}$ & 963 & $\begin{array}{l}402.02 \\
(11.09)\end{array}$ & $\begin{array}{c}295.09 \\
(0.00 \\
2801.47)\end{array}$ & 597 & $\begin{array}{l}412.67 \\
(12.52)\end{array}$ & $\begin{array}{c}318.32 \\
(0.00 \\
1822.92)\end{array}$ & 225 & $\begin{array}{l}339.64 \\
(16.42)\end{array}$ & $\begin{array}{c}268.78 \\
(0.00 \\
1545.11)\end{array}$ \\
\hline Men & 772 & $\begin{array}{l}410.55 \\
(11.81)\end{array}$ & $\begin{array}{c}305.19 \\
(0.00 \\
2801.47)\end{array}$ & 30 & $\begin{array}{l}375.82 \\
(44.50)\end{array}$ & $\begin{array}{c}326.02 \\
(0.00 \\
951.57)\end{array}$ & 384 & $\begin{array}{l}408.13 \\
(18.20)\end{array}$ & $\begin{array}{c}302.11 \\
(0.00 \\
2801.47)\end{array}$ & 265 & $\begin{array}{l}430.97 \\
(19.18)\end{array}$ & $\begin{array}{c}308.70 \\
(0.60 \\
1822.92)\end{array}$ & 93 & $\begin{array}{l}373.56 \\
(27.59)\end{array}$ & $\begin{array}{c}289.75 \\
(18.08 \\
1207.35)\end{array}$ \\
\hline Women & 1066 & $\begin{array}{c}385.33 \\
(9.54)\end{array}$ & $\begin{array}{c}285.57 \\
(0.00 \\
2305.08)\end{array}$ & 23 & $\begin{array}{l}282.93 \\
(25.86)\end{array}$ & $\begin{array}{c}257.78 \\
(72,56 \\
541.09)\end{array}$ & 579 & $\begin{array}{l}397.96 \\
(13.95)\end{array}$ & $\begin{array}{c}291.35 \\
(0.00 \\
2305.08)\end{array}$ & 332 & $\begin{array}{l}398.06 \\
(16.50)\end{array}$ & $\begin{array}{c}320.91 \\
(0.00 \\
1791.90)\end{array}$ & 132 & $\begin{array}{l}315.74 \\
(19.98)\end{array}$ & $\begin{array}{c}253.81 \\
(0.00 \\
1545.11)\end{array}$ \\
\hline \multicolumn{16}{|c|}{$\begin{array}{l}\text { Non-starchy } \\
\text { vegetables }\end{array}$} \\
\hline Total & 1838 & $\begin{array}{c}219.48 \\
(3.22)\end{array}$ & $\begin{array}{c}195.86 \\
(0.00 \\
1506.75)\end{array}$ & 53 & $\begin{array}{l}250.47 \\
(28.28)\end{array}$ & $\begin{array}{c}192.78 \\
(1.13 \\
1236.37)\end{array}$ & 963 & $\begin{array}{c}214.51 \\
(4.54)\end{array}$ & $\begin{array}{c}189.54 \\
(0.00 \\
1506.75)\end{array}$ & 597 & $\begin{array}{c}222.71 \\
(5.29)\end{array}$ & $\begin{array}{c}199.63 \\
(0.00 \\
1254.12)\end{array}$ & 225 & $\begin{array}{l}224.87 \\
(8.58)\end{array}$ & $\begin{array}{c}217.68 \\
(0.00 \\
1268.28)\end{array}$ \\
\hline Men & 772 & $\begin{array}{c}221.43 \\
(5.04)\end{array}$ & $\begin{array}{c}195.80 \\
(0.00 \\
1506.75)\end{array}$ & 30 & $\begin{array}{l}254.14 \\
(31.47)\end{array}$ & $\begin{array}{c}204.14 \\
(1.13, \\
799.94)\end{array}$ & 384 & $\begin{array}{c}211.29 \\
(7.58)\end{array}$ & $\begin{array}{c}182.75 \\
(0.00 \\
1506.75)\end{array}$ & 265 & $\begin{array}{c}227.06 \\
(7.88)\end{array}$ & $\begin{array}{c}203.94 \\
(1.50 \\
709.37)\end{array}$ & 93 & $\begin{array}{l}236.69 \\
(12.51)\end{array}$ & $\begin{array}{l}235.67 \\
(36.69 \\
567.68)\end{array}$ \\
\hline Women & 1066 & $\begin{array}{l}218.07 \\
(4.19)\end{array}$ & $\begin{array}{c}195.86 \\
(0.00 \\
1268.28)\end{array}$ & 23 & $\begin{array}{l}245.68 \\
(51.48)\end{array}$ & $\begin{array}{c}183.20 \\
(33.78, \\
1236.37)\end{array}$ & 579 & $\begin{array}{c}216.65 \\
(5.63)\end{array}$ & $\begin{array}{c}192.75 \\
(0.00 \\
1146.28)\end{array}$ & 332 & $\begin{array}{c}219.23 \\
(7.15)\end{array}$ & $\begin{array}{c}197.86 \\
(0.00 \\
1254.12)\end{array}$ & 132 & $\begin{array}{l}216.54 \\
(11.56)\end{array}$ & $\begin{array}{c}208.32 \\
(0.0 \\
1268.28)\end{array}$ \\
\hline
\end{tabular}


Table 3. Cont.

\begin{tabular}{|c|c|c|c|c|c|c|c|c|c|c|c|c|c|c|c|}
\hline & \multicolumn{3}{|c|}{ Total } & \multicolumn{3}{|c|}{$<20$ years } & \multicolumn{3}{|c|}{$20<$ years $<50$} & \multicolumn{3}{|c|}{$50<$ years $<70$} & \multicolumn{3}{|c|}{$>70$ years } \\
\hline & $n$ & $\begin{array}{l}\text { Mean } \\
\text { (SE) }\end{array}$ & $\begin{array}{l}\text { Median } \\
\text { (Range) }\end{array}$ & $n$ & $\begin{array}{l}\text { Mean } \\
\text { (SE) }\end{array}$ & $\begin{array}{l}\text { Median } \\
\text { (Range) }\end{array}$ & $n$ & $\begin{array}{l}\text { Mean } \\
\text { (SE) }\end{array}$ & $\begin{array}{l}\text { Median } \\
\text { (Range) }\end{array}$ & $n$ & $\begin{array}{l}\text { Mean } \\
\text { (SE) }\end{array}$ & $\begin{array}{l}\text { Median } \\
\text { (Range) }\end{array}$ & $n$ & $\begin{array}{l}\text { Mean } \\
\text { (SE) }\end{array}$ & $\begin{array}{l}\text { Median } \\
\text { (Range) }\end{array}$ \\
\hline \multicolumn{16}{|c|}{$\begin{array}{l}\text { Other starchy } \\
\text { vegetables }\end{array}$} \\
\hline Total & 1838 & $\begin{array}{l}16.33 \\
(0.44)\end{array}$ & $\begin{array}{c}14.00 \\
(0.00 \\
450.90)\end{array}$ & 53 & $\begin{array}{l}15.88 \\
(2.06)\end{array}$ & $\begin{array}{l}14.00 \\
(0.00 \\
66.00)\end{array}$ & 963 & $\begin{array}{l}17.24 \\
(0.72)\end{array}$ & $\begin{array}{c}14.00 \\
(0.00 \\
450.90)\end{array}$ & 597 & $\begin{array}{l}15.22 \\
(0.57)\end{array}$ & $\begin{array}{c}14.00 \\
(0.00 \\
130.00)\end{array}$ & 225 & $\begin{array}{l}15.46 \\
(0.87)\end{array}$ & $\begin{array}{l}14.00 \\
(0.00 \\
66.00)\end{array}$ \\
\hline Men & 772 & $\begin{array}{l}17.07 \\
(0.80)\end{array}$ & $\begin{array}{c}14.00 \\
(0.00 \\
450.90)\end{array}$ & 30 & $\begin{array}{l}17.08 \\
(2.94)\end{array}$ & $\begin{array}{l}14.45 \\
(0.00 \\
66.00)\end{array}$ & 384 & $\begin{array}{l}18.04 \\
(1.43)\end{array}$ & $\begin{array}{c}14.00 \\
(0.00 \\
450.90)\end{array}$ & 265 & $\begin{array}{l}16.03 \\
(0.93)\end{array}$ & $\begin{array}{c}14.00 \\
(0.00 \\
130.00)\end{array}$ & 93 & $\begin{array}{l}16.00 \\
(1.34)\end{array}$ & $\begin{array}{l}14.00 \\
(0.00 \\
66.00)\end{array}$ \\
\hline Women & 1066 & $\begin{array}{l}15.80 \\
(0.48)\end{array}$ & $\begin{array}{c}14.00 \\
(0.00 \\
130.00)\end{array}$ & 23 & $\begin{array}{l}14.31 \\
(2.85)\end{array}$ & $\begin{array}{c}8.71 \\
(0.00 \\
46.80)\end{array}$ & 579 & $\begin{array}{l}16.72 \\
(0.73)\end{array}$ & $\begin{array}{c}14.00 \\
(0.00 \\
130.00)\end{array}$ & 332 & $\begin{array}{l}14.57 \\
(0.72)\end{array}$ & $\begin{array}{l}14.00 \\
(0.00 \\
74.80)\end{array}$ & 132 & $\begin{array}{l}15.08 \\
(1.14)\end{array}$ & $\begin{array}{l}14.00 \\
(0.00 \\
66.00)\end{array}$ \\
\hline \multicolumn{16}{|l|}{$\begin{array}{l}\text { Beans and } \\
\text { legumes }\end{array}$} \\
\hline Total & 1838 & $\begin{array}{l}35.61 \\
(0.88)\end{array}$ & $\begin{array}{c}23.70 \\
(0.00 \\
655.33)\end{array}$ & 53 & $\begin{array}{l}35.35 \\
(4.78)\end{array}$ & $\begin{array}{r}23.10 \\
(0.00 \\
130.23) \\
\end{array}$ & 963 & $\begin{array}{l}36.69 \\
(1.32)\end{array}$ & $\begin{array}{c}24.00 \\
(0.00 \\
655.33) \\
\end{array}$ & 597 & $\begin{array}{l}33.69 \\
(1.33)\end{array}$ & $\begin{array}{c}23.70 \\
(0.00 \\
325.33)\end{array}$ & 225 & $\begin{array}{l}36.15 \\
(2.48)\end{array}$ & $\begin{array}{c}22.33 \\
(0.00 \\
184.00) \\
\end{array}$ \\
\hline Men & 772 & $\begin{array}{l}36.33 \\
(1.48)\end{array}$ & $\begin{array}{c}23.85 \\
(0.00 \\
655.33)\end{array}$ & 30 & $\begin{array}{l}36.18 \\
(6.39)\end{array}$ & $\begin{array}{r}27.17 \\
(0.00 \\
129.00) \\
\end{array}$ & 384 & $\begin{array}{l}37.19 \\
(2.33)\end{array}$ & $\begin{array}{c}24.10 \\
(0.00 \\
655.33)\end{array}$ & 265 & $\begin{array}{l}34.65 \\
(2.15)\end{array}$ & $\begin{array}{c}24.70 \\
(0.00 \\
325.33)\end{array}$ & 93 & $\begin{array}{l}37.58 \\
(4.09)\end{array}$ & $\begin{array}{c}22.33 \\
(3.00 \\
179.00) \\
\end{array}$ \\
\hline Women & 1066 & $\begin{array}{l}35.09 \\
(1.08)\end{array}$ & $\begin{array}{c}23.40 \\
(0.00 \\
210.70)\end{array}$ & 23 & $\begin{array}{l}34.27 \\
(7.35)\end{array}$ & $\begin{array}{r}22.33 \\
(5.23 \\
130.23)\end{array}$ & 579 & $\begin{array}{l}36.36 \\
(1.56)\end{array}$ & $\begin{array}{c}23.70 \\
(0.00 \\
210.70)\end{array}$ & 332 & $\begin{array}{l}32.91 \\
(1.67)\end{array}$ & $\begin{array}{c}23.40 \\
(0.00 \\
210.70)\end{array}$ & 132 & $\begin{array}{l}35.14 \\
(3.10)\end{array}$ & $\begin{array}{c}22.28 \\
(0.00 \\
184.00)\end{array}$ \\
\hline
\end{tabular}


Table 3. Cont.

\begin{tabular}{|c|c|c|c|c|c|c|c|c|c|c|c|c|c|c|c|}
\hline & \multicolumn{3}{|c|}{ Total } & \multicolumn{3}{|c|}{$<20$ years } & \multicolumn{3}{|c|}{$20<$ years $<50$} & \multicolumn{3}{|c|}{$50<$ years $<70$} & \multicolumn{3}{|c|}{$>70$ years } \\
\hline & $n$ & $\begin{array}{l}\text { Mean } \\
\text { (SE) }\end{array}$ & $\begin{array}{l}\text { Median } \\
\text { (Range) }\end{array}$ & $n$ & $\begin{array}{l}\text { Mean } \\
\text { (SE) }\end{array}$ & $\begin{array}{l}\text { Median } \\
\text { (Range) }\end{array}$ & $n$ & $\begin{array}{l}\text { Mean } \\
\text { (SE) }\end{array}$ & $\begin{array}{l}\text { Median } \\
\text { (Range) }\end{array}$ & $n$ & $\begin{array}{l}\text { Mean } \\
\text { (SE) }\end{array}$ & $\begin{array}{l}\text { Median } \\
\text { (Range) }\end{array}$ & $n$ & $\begin{array}{l}\text { Mean } \\
\text { (SE) }\end{array}$ & $\begin{array}{l}\text { Median } \\
\text { (Range) }\end{array}$ \\
\hline \multicolumn{16}{|l|}{$\begin{array}{l}\text { Nuts and } \\
\text { seeds }\end{array}$} \\
\hline Total & 1838 & $\begin{array}{l}20.30 \\
(0.73)\end{array}$ & $\begin{array}{c}11.52 \\
(0.00 \\
408.40)\end{array}$ & 53 & $\begin{array}{l}19.77 \\
(4.48)\end{array}$ & $\begin{array}{c}9.05 \\
(0.00 \\
190.00)\end{array}$ & 963 & $\begin{array}{l}19.87 \\
(0.98)\end{array}$ & $\begin{array}{c}10.35 \\
(0.00 \\
408.40)\end{array}$ & 597 & $\begin{array}{l}21.89 \\
(1.42)\end{array}$ & $\begin{array}{c}12.75 \\
(0.00 \\
408.40)\end{array}$ & 225 & $\begin{array}{l}18.10 \\
(1.60)\end{array}$ & $\begin{array}{c}10.05 \\
(0.00 \\
153.40)\end{array}$ \\
\hline Men & 772 & $\begin{array}{l}20.91 \\
(1.21)\end{array}$ & $\begin{array}{c}10.40 \\
(0.00 \\
408.40)\end{array}$ & 30 & $\begin{array}{l}23.29 \\
(7.54)\end{array}$ & $\begin{array}{c}7.71 \\
(0.00 \\
190.00)\end{array}$ & 384 & $\begin{array}{l}19.42 \\
(1.92)\end{array}$ & $\begin{array}{c}7.94 \\
(0.00 \\
408.40)\end{array}$ & 265 & $\begin{array}{l}22.69 \\
(1.70)\end{array}$ & $\begin{array}{c}13.40 \\
(0.00 \\
190.00)\end{array}$ & 93 & $\begin{array}{l}21.20 \\
(3.05)\end{array}$ & $\begin{array}{c}10.35 \\
(0.00 \\
153.40)\end{array}$ \\
\hline Women & 1066 & $\begin{array}{l}19.87 \\
(0.90)\end{array}$ & $\begin{array}{c}11.70 \\
(0.00 \\
408.40)\end{array}$ & 23 & $\begin{array}{l}15.17 \\
(3.16)\end{array}$ & $\begin{array}{l}11.70 \\
(0.00 \\
68.80)\end{array}$ & 579 & $\begin{array}{l}20.16 \\
(1.02)\end{array}$ & $\begin{array}{c}12.73 \\
(0.00 \\
190.00)\end{array}$ & 332 & $\begin{array}{l}21.26 \\
(2.17)\end{array}$ & $\begin{array}{c}11.52 \\
(0.00 \\
408.40)\end{array}$ & 132 & $\begin{array}{l}15.91 \\
(1.66)\end{array}$ & $\begin{array}{c}9.92 \\
(0.00 \\
101.48) \\
\end{array}$ \\
\hline \multicolumn{16}{|l|}{ Potatoes } \\
\hline Total & 1838 & $\begin{array}{l}25.52 \\
(0.58)\end{array}$ & $\begin{array}{c}17.75 \\
(0.00 \\
450.75)\end{array}$ & 53 & $\begin{array}{l}31.09 \\
(5.13)\end{array}$ & $\begin{array}{c}24.20 \\
(0.00 \\
253.00)\end{array}$ & 963 & $\begin{array}{l}25.86 \\
(0.85)\end{array}$ & $\begin{array}{c}17.75 \\
(0.00 \\
450.75)\end{array}$ & 597 & $\begin{array}{l}24.98 \\
(0.95)\end{array}$ & $\begin{array}{c}17.00 \\
(0.00 \\
169.20)\end{array}$ & 225 & $\begin{array}{l}24.21 \\
(1.30)\end{array}$ & $\begin{array}{c}17.00 \\
(0.00 \\
106.70)\end{array}$ \\
\hline Men & 772 & $\begin{array}{l}26.48 \\
(0.87)\end{array}$ & $\begin{array}{c}17.75 \\
(0.00 \\
180.00)\end{array}$ & 30 & $\begin{array}{l}30.72 \\
(4.27)\end{array}$ & $\begin{array}{c}24.20 \\
(0.00 \\
100.00)\end{array}$ & 384 & $\begin{array}{l}26.17 \\
(1.22)\end{array}$ & $\begin{array}{c}17.50 \\
(0.00 \\
180.00)\end{array}$ & 265 & $\begin{array}{l}26.56 \\
(1.61)\end{array}$ & $\begin{array}{c}17.00 \\
(0.00 \\
169.20)\end{array}$ & 93 & $\begin{array}{l}26.23 \\
(2.06)\end{array}$ & $\begin{array}{c}20.70 \\
(0.00 \\
103.00)\end{array}$ \\
\hline Women & 1066 & $\begin{array}{l}24.82 \\
(0.78)\end{array}$ & $\begin{array}{c}17.50 \\
(0.00 \\
450.75)\end{array}$ & 23 & $\begin{array}{c}31.56 \\
(10.58)\end{array}$ & $\begin{array}{c}20.70 \\
(0.00 \\
253.00)\end{array}$ & 579 & $\begin{array}{l}25.65 \\
(1.16)\end{array}$ & $\begin{array}{c}18.68 \\
(0.00 \\
450.75)\end{array}$ & 332 & $\begin{array}{l}23.73 \\
(1.12)\end{array}$ & $\begin{array}{c}17.00 \\
(0.00 \\
136.00)\end{array}$ & 132 & $\begin{array}{l}22.78 \\
(1.68)\end{array}$ & $\begin{array}{c}16.34 \\
(0.00, \\
106.70)\end{array}$ \\
\hline
\end{tabular}


Table 3. Cont.

\begin{tabular}{|c|c|c|c|c|c|c|c|c|c|c|c|c|c|c|c|}
\hline & \multicolumn{3}{|c|}{ Total } & \multicolumn{3}{|c|}{$<20$ years } & \multicolumn{3}{|c|}{$20<$ years $<50$} & \multicolumn{3}{|c|}{$50<$ years $<70$} & \multicolumn{3}{|c|}{$>70$ years } \\
\hline & $n$ & $\begin{array}{l}\text { Mean } \\
\text { (SE) }\end{array}$ & $\begin{array}{l}\text { Median } \\
\text { (Range) }\end{array}$ & $n$ & $\begin{array}{l}\text { Mean } \\
\text { (SE) }\end{array}$ & $\begin{array}{l}\text { Median } \\
\text { (Range) }\end{array}$ & $n$ & $\begin{array}{l}\text { Mean } \\
\text { (SE) }\end{array}$ & $\begin{array}{l}\text { Median } \\
\text { (Range) }\end{array}$ & $n$ & $\begin{array}{l}\text { Mean } \\
\text { (SE) }\end{array}$ & $\begin{array}{l}\text { Median } \\
\text { (Range) }\end{array}$ & $n$ & $\begin{array}{l}\text { Mean } \\
\text { (SE) }\end{array}$ & $\begin{array}{l}\text { Median } \\
\text { (Range) }\end{array}$ \\
\hline \multicolumn{16}{|c|}{ Whole grains } \\
\hline Total & 1838 & $\begin{array}{l}27.38 \\
(1.19)\end{array}$ & $\begin{array}{c}3.00 \\
(0.00 \\
330.00)\end{array}$ & 53 & $\begin{array}{l}26.21 \\
(5.42)\end{array}$ & $\begin{array}{c}1.01 \\
(0.00 \\
151.20)\end{array}$ & 963 & $\begin{array}{l}29.34 \\
(1.74)\end{array}$ & $\begin{array}{c}3.00 \\
(0.00 \\
330.00)\end{array}$ & 597 & $\begin{array}{l}25.90 \\
(1.99)\end{array}$ & $\begin{array}{c}3.00 \\
(0.00 \\
298.70)\end{array}$ & 225 & $\begin{array}{l}23.21 \\
(3.01)\end{array}$ & $\begin{array}{c}2.10 \\
(0.00 \\
270.36)\end{array}$ \\
\hline Men & 772 & $\begin{array}{l}26.56 \\
(1.77)\end{array}$ & $\begin{array}{c}3.00 \\
(0.00 \\
330.00)\end{array}$ & 30 & $\begin{array}{l}37.73 \\
(8.69)\end{array}$ & $\begin{array}{c}9.00 \\
(0.00 \\
151.20)\end{array}$ & 384 & $\begin{array}{l}30.52 \\
(2.78)\end{array}$ & $\begin{array}{c}5.40 \\
(0.00 \\
330.00)\end{array}$ & 265 & $\begin{array}{l}20.54 \\
(2.58)\end{array}$ & $\begin{array}{c}3.00 \\
(0.00 \\
298.70)\end{array}$ & 93 & $\begin{array}{l}23.75 \\
(4.59)\end{array}$ & $\begin{array}{c}3.00 \\
(0.00 \\
252.85)\end{array}$ \\
\hline Women & 1066 & $\begin{array}{l}27.97 \\
(1.59)\end{array}$ & $\begin{array}{c}2.10 \\
(0.00 \\
330.00)\end{array}$ & 23 & $\begin{array}{c}11.20 \\
3.50\end{array}$ & $\begin{array}{c}0.45 \\
(0.00 \\
46.80)\end{array}$ & 579 & $\begin{array}{l}28.55 \\
(2.23)\end{array}$ & $\begin{array}{c}3.00 \\
(0.00 \\
330.00)\end{array}$ & 332 & $\begin{array}{l}30.17 \\
(2.91)\end{array}$ & $\begin{array}{c}3.00 \\
(0.00 \\
298.70)\end{array}$ & 132 & $\begin{array}{l}22.84 \\
(4.00)\end{array}$ & $\begin{array}{c}0.73 \\
(0.00 \\
270.36)\end{array}$ \\
\hline \multicolumn{16}{|l|}{$\begin{array}{l}\text { Refined } \\
\text { grains }\end{array}$} \\
\hline Total & 1838 & $\begin{array}{l}214.10 \\
(3.04)\end{array}$ & $\begin{array}{c}184.15 \\
(3.00 \\
909.26)\end{array}$ & 53 & $\begin{array}{l}197.89 \\
(17.80)\end{array}$ & $\begin{array}{c}174.05 \\
(3.00 \\
576.71)\end{array}$ & 963 & $\begin{array}{c}210.54 \\
(4.15)\end{array}$ & $\begin{array}{c}180.89 \\
(4.50 \\
909.26)\end{array}$ & 597 & $\begin{array}{c}220.89 \\
(5.51)\end{array}$ & $\begin{array}{c}189.00 \\
(6.70 \\
909.26)\end{array}$ & 225 & $\begin{array}{l}215.19 \\
(8.41)\end{array}$ & $\begin{array}{l}185.05 \\
(11.28 \\
630.03)\end{array}$ \\
\hline Men & 772 & $\begin{array}{c}217.24 \\
(4.60)\end{array}$ & $\begin{array}{c}187.39 \\
(3.00 \\
909.26)\end{array}$ & 30 & $\begin{array}{l}169.00 \\
(20.22)\end{array}$ & $\begin{array}{c}173.83 \\
(3.00 \\
420.10)\end{array}$ & 384 & $\begin{array}{c}217.19 \\
(6.47)\end{array}$ & $\begin{array}{c}186.51 \\
(12.60 \\
589.85)\end{array}$ & 265 & $\begin{array}{l}226.87 \\
(8.25)\end{array}$ & $\begin{array}{c}196.70 \\
(11.28 \\
909.26)\end{array}$ & 93 & $\begin{array}{l}205.57 \\
(11.86)\end{array}$ & $\begin{array}{l}180.31 \\
(25.20, \\
541.06)\end{array}$ \\
\hline Women & 1066 & $\begin{array}{c}211.83 \\
(4.06)\end{array}$ & $\begin{array}{c}182.40 \\
(4.50 \\
909.26)\end{array}$ & 23 & $\begin{array}{l}235.58 \\
(30.14)\end{array}$ & $\begin{array}{c}173.83 \\
(3.00 \\
420.10)\end{array}$ & 579 & $\begin{array}{c}206.12 \\
(5.41)\end{array}$ & $\begin{array}{c}179.66 \\
(4.50 \\
909.26)\end{array}$ & 332 & $\begin{array}{c}216.12 \\
(7.41)\end{array}$ & $\begin{array}{c}182.60 \\
(6.70 \\
696.60)\end{array}$ & 132 & $\begin{array}{l}221.98 \\
(11.65)\end{array}$ & $\begin{array}{l}187.62 \\
(11.28, \\
630.03)\end{array}$ \\
\hline
\end{tabular}


Table 4. Pearson/Spearman correlation coefficients between major food groups intake. ${ }^{*}$ denotes $p<0.05,{ }^{* *}$ denotes $p<0.001$.

\begin{tabular}{|c|c|c|c|c|c|c|c|c|c|c|c|c|c|c|}
\hline & $\begin{array}{c}\text { Total } \\
\text { Processed } \\
\text { Meats }\end{array}$ & $\begin{array}{l}\text { Unprocessed } \\
\text { Red Meats }\end{array}$ & $\begin{array}{c}\text { Total } \\
\text { Seafood }\end{array}$ & Eggs & Cheese & Yoghurt & Fruits & $\begin{array}{l}\text { Non-Starchy } \\
\text { Vegetables }\end{array}$ & Potatoes & $\begin{array}{c}\text { Other } \\
\text { Starchy } \\
\text { Vegetables }\end{array}$ & $\begin{array}{c}\text { Beans } \\
\text { and } \\
\text { Legumes }\end{array}$ & $\begin{array}{l}\text { Nuts } \\
\text { and } \\
\text { Seeds }\end{array}$ & $\begin{array}{c}\text { Refined } \\
\text { Grains }\end{array}$ & $\begin{array}{l}\text { Whole } \\
\text { Grains }\end{array}$ \\
\hline $\begin{array}{c}\text { Total } \\
\text { processed } \\
\text { meats }\end{array}$ & 1 & - & - & - & - & - & - & - & - & - & - & - & - & - \\
\hline $\begin{array}{l}\text { Unprocessed } \\
\text { red meats }\end{array}$ & $0.217^{* *}$ & 1 & - & - & - & - & - & - & - & - & - & - & - & - \\
\hline $\begin{array}{c}\text { Total } \\
\text { seafood }\end{array}$ & $0.162 * *$ & $0.072 * *$ & 1 & - & - & - & - & - & - & - & - & - & - & - \\
\hline Eggs & 0.003 & $0.179 * *$ & $0.093 * *$ & 1 & - & - & - & - & - & - & - & - & - & - \\
\hline Cheese & $0.251^{* *}$ & $0.200 * *$ & $0.189 * *$ & $0.094^{* *}$ & 1 & - & - & - & - & - & - & - & - & - \\
\hline Yoghurt & -0.010 & -0.032 & $0.145^{* *}$ & 0.034 & 0.0123 ** & 1 & - & - & - & - & - & - & - & - \\
\hline Fruits & 0.004 & -0.004 & $0.121 * *$ & -0.010 & $0.074^{* *}$ & $0.113^{* *}$ & 1 & & - & - & - & - & - & - \\
\hline $\begin{array}{l}\text { Non-starchy } \\
\text { vegetables }\end{array}$ & -0.002 & -0.037 & $0.209 * *$ & 0.016 & $0.167^{* *}$ & $0.138^{* *}$ & $0.297^{* *}$ & 1 & - & - & - & - & - & - \\
\hline Potatoes & $0.316^{* *}$ & $0.085^{* *}$ & $0.151 * *$ & $0.084^{* *}$ & 0.305 ** & 0.063 ** & $0.073 * *$ & $0.075^{* *}$ & 1 & - & - & - & - & - \\
\hline $\begin{array}{c}\text { Other } \\
\text { starchy } \\
\text { vegetables }\end{array}$ & $0.075^{* *}$ & -0.029 & 0.203 ** & 0.018 & $0.138^{* *}$ & $0.073^{* *}$ & $0.258^{* *}$ & $0.399 * *$ & $0.144^{* *}$ & 1 & - & - & - & - \\
\hline $\begin{array}{c}\text { Beans and } \\
\text { legumes }\end{array}$ & 0.044 & 0.000 & $0.268^{* *}$ & 0.041 & $0.108^{* *}$ & $0.114^{* *}$ & $0.203 * *$ & $0.370 * *$ & $0.052 *$ & $0.211^{* *}$ & 1 & - & - & - \\
\hline $\begin{array}{l}\text { Nuts and } \\
\text { seeds }\end{array}$ & $0.098^{* *}$ & $0.069^{* *}$ & $0.048^{*}$ & 0.036 & $0.084^{* *}$ & 0.005 & -0.037 & 0.060 ** & 0.080 ** & -0.002 & $0.071^{* *}$ & 1 & - & - \\
\hline $\begin{array}{c}\text { Refined } \\
\text { grains }\end{array}$ & 0.052 * & $0.189 * *$ & $-0.055^{*}$ & $0.154^{* *}$ & $0.197^{* *}$ & $-\underset{* *}{-0.152}$ & 0.034 & -0.029 & $0.055^{*}$ & -0.032 & -0.017 & -0.021 & 1 & - \\
\hline $\begin{array}{l}\text { Whole } \\
\text { grains }\end{array}$ & $0.058^{*}$ & $-0.057^{*}$ & $0.109 * *$ & $\underset{* *}{-0.065}$ & $0.079^{* *}$ & $0.190^{* *}$ & $0.151^{* *}$ & $0.196^{* *}$ & 0.008 & $0.070^{* *}$ & $0.090^{* *}$ & -0.042 & $\underset{* *}{-0.119}$ & 1 \\
\hline
\end{tabular}


Table 5. Percentage of study population meeting various recommendations for macronutrients (EFSA, LARN) and selected food groups (WHO).

\begin{tabular}{|c|c|c|c|c|c|c|}
\hline & \multicolumn{2}{|c|}{ Total $(n=1839)$} & \multicolumn{2}{|c|}{ Men $(n=772)$} & \multicolumn{2}{|c|}{ Women $(n=1066)$} \\
\hline & Yes, \% (n) & No, $\%(n)$ & Yes, \% (n) & No, $\%(n)$ & Yes, \% (n) & No, $\%(n)$ \\
\hline \multicolumn{7}{|l|}{ EFSA } \\
\hline Total carbohydrate (45-60\%E) & $56.3(1035)$ & 43.7 (804) & $56.6(437)$ & $43.4(335)$ & $56.0(597)$ & $44.0(469)$ \\
\hline Total protein $(>0.83 \mathrm{~g} / \mathrm{kg} /$ day $)$ & $89.7(1649)$ & $10.3(190)$ & $85.5(660)$ & $14.5(112)$ & $92.8(989)$ & $7.2(77)$ \\
\hline Total fat $(20-35 \% \mathrm{E})$ & $17.1(315)$ & $82.9(1524)$ & $17.6(136)$ & $82.4(636)$ & $16.8(179)$ & $83.2(887)$ \\
\hline Fiber (>25 g/day) & $62.8(1154)$ & $37.2(685)$ & $62.7(484)$ & $37.3(288)$ & $62.9(670)$ & $37.1(396)$ \\
\hline \multicolumn{7}{|l|}{ LARN } \\
\hline Total carbohydrates $(40-60 \% \mathrm{E})$ & 59.4 (1092) & 40.6 (747) & $59.5(459)$ & 40.5 (313) & $59.3(632)$ & 40.7 (434) \\
\hline Total protein $(>0.90 \mathrm{~g} / \mathrm{kg} /$ day $)$ & $83.9(1543)$ & $16.1(296)$ & $78.6(607)$ & $21.4(165)$ & $87.8(936)$ & $12.2(130)$ \\
\hline Total fat $(20-35 \% \mathrm{E})$ & $17.1(315)$ & $82.9(1524)$ & $17.6(136)$ & $82.4(636)$ & $16.8(179)$ & $83.2(887)$ \\
\hline Cholesterol (<300 mg/day) & $91.8(1688)$ & $8.2(151)$ & $91.3(705)$ & $8.7(67)$ & $92.1(982)$ & $7.9(84)$ \\
\hline $\begin{array}{l}\text { Fiber (12.6-16.7 g/1000 } \\
\text { kcal/day) }\end{array}$ & $53.5(983)$ & $46.5(856)$ & $54.3(419)$ & $45.7(353)$ & $52.9(564)$ & $47.1(502)$ \\
\hline \multicolumn{7}{|l|}{ WHO } \\
\hline Fruit and vegetable (>400 g/day) & $74.6(1371)$ & $25.4(468)$ & $76.4(590)$ & $23.6(182)$ & $73.2(780)$ & $26.8(286)$ \\
\hline Pulses and nuts (>30 g/day) & $81.7(1503)$ & $18.3(336)$ & $82.5(637)$ & $17.5(135)$ & $81.2(866)$ & $18.8(200)$ \\
\hline Total meat $(<70 \mathrm{~g} /$ day $)$ & $77.0(1416)$ & $23.0(423)$ & $75.4(582)$ & $24.6(190)$ & $78.1(833)$ & $21.9(233)$ \\
\hline
\end{tabular}

Table 6. Linear association between macronutrient intake and BMI levels in the study participants of the MEAL study $(n=1838)$. ${ }^{*}$ denotes $p<0.05,{ }^{* *}$ denotes $p<0.001$.

\begin{tabular}{lccc}
\hline & Total & Men & Women \\
\hline Total carbohydrates & $0.000(0.006)$ & $-0.002(0.008)$ & $-0.001(0.008)$ \\
Total protein & $-0.035(0.016)^{*}$ & $-0.022(0.025)$ & $-0.046(0.022)^{*}$ \\
Animal protein & $0.000(0.006)$ & $-0.002(0.010)$ & $0.002(0.008)$ \\
Dairy protein & $-0.013(0.013)$ & $0.002(0.020)$ & $-0.023(0.018)$ \\
Plant protein & $-0.049(0.022)^{*}$ & $0.010(0.033)$ & $-0.081(0.031)^{* *}$ \\
Saturated fat & $-0.023(0.041)$ & $-0.108(0.064)$ & $0.028(0.053)$ \\
Monounsaturated fat & $-0.707(0.138)^{* *}$ & $-0.594(0.212)^{* *}$ & $-0.838(0.184)^{* *}$ \\
Total omega-6 fatty acids & $-0.657(0.173)^{* *}$ & $-0.722(0.251)^{* *}$ & $-0.647(0.242)^{* *}$ \\
Seafood omega-3 fat & $0.024(0.356)$ & $0.272(0.548)$ & $-0.204(0.471)$ \\
Plant omega-3 fat & $0.654(0.522)$ & $2.178(0.879)^{*}$ & $-0.213(0.748)$ \\
Trans fatty acid & $0.666(0.135)^{* *}$ & $0.520(0.202)^{*}$ & $0.807(0.184)^{* *}$ \\
Dietary cholesterol & $0.021(0.004)^{* *}$ & $0.015(0.007)^{*}$ & $0.026(0.006)^{* *}$ \\
Fiber & $-0.013(0.016)$ & $-0.054(0.024)^{*}$ & $0.014(0.021)$ \\
\hline
\end{tabular}

Table 7. Linear association between major food group intake and BMI levels in the study participants of the MEAL study $(n=1838)$. ${ }^{*}$ denotes $p<0.05,{ }^{* *}$ denotes $p<0.001$.

\begin{tabular}{lccc}
\hline & Total & Men & Women \\
\hline Total processed meats & $-0.023(0.007)^{* *}$ & $-0.031(0.010)^{* *}$ & $-0.015(0.010)$ \\
Unprocessed meats & $0.017(0.005)^{* *}$ & $0.014(0.007)^{*}$ & $0.019(0.006)^{* *}$ \\
Total seafood & $0.004(0.002)$ & $0.007(0.003)^{*}$ & $0.002(0.003)$ \\
Eggs & $0.039(0.024)$ & $0.037(0.043)$ & $0.042(0.031)$ \\
Cheese & $0.004(0.004)$ & $-0.001(0.007)$ & $0.008(0.006)$ \\
Yoghurt & $-0.002(0.003)$ & $-0.010(0.004)^{*}$ & $0.003(0.003)$ \\
Fruits & $-0.001(0.000)$ & $-0.001(0.001)$ & $-0.001(0.001)$ \\
Non-starchy vegetables & $0.001(0.001)$ & $0.002(0.002)$ & $0.001(0.001)$ \\
Potatoes & $-0.005(0.005)$ & $-0.003(0.009)$ & $-0.007(0.006)$ \\
Other starchy vegetables & $0.002(0.007)$ & $0.004(0.009)$ & $-0.005(0.011)$ \\
Beans and legumes & $0.001(0.003)$ & $-0.008(0.005)$ & $0.007(0.005)$ \\
Nuts and seeds & $0.002(0.004)$ & $0.002(0.006)$ & $0.000(0.006)$ \\
Refined grains & $0.002(0.002)$ & $0.001(0.002)$ & $0.002(0.002)$ \\
Whole grains & $-0.004(0.003)$ & $-0.004(0.004)$ & $-0.003(0.003)$ \\
\hline
\end{tabular}




\section{Discussion}

The present study provided updated information on intake of major food groups and macronutrients and their association with weight status in a sample of southern Italian adults. We found that a large proportion of individuals had adequate intake of protein, fiber, fats, fruit and vegetable, meat, and pulses according to national and international recommendations. These results suggest that the investigated population has generally healthy dietary choices; however, investigating major food group consumption and comparison with other reports is crucial to better understand dietary priorities for future strategies to improve dietary habits and overall health.

Despite the importance of monitoring dietary intakes at population level, previous studies investigating macronutrient and food consumption are scarce. A recent report of Global Burden of Diseases Nutrition and Chronic Diseases Expert Group aimed to describe consumption of major food groups worldwide and at national level [31]. Despite that the report showed standardized intake to the same isocaloric diet $(2000 \mathrm{kcal} /$ day $)$, our data are comparable, due to similar average total energy intake in both men and women. In 2010, mean global fruit consumption in adults has been reported to be $81.3 \mathrm{~g} /$ day, with the highest intake in Greece, and no clear pattern of variation of consumption worldwide. In this study, we reported a much higher fruit intake (about $400 \mathrm{~g} /$ day) only comparable with reports from Jamaica and Malaysia. However, two Italian surveys [32,33] showed an average national consumption of fruit closer to those reported in the present study (about 200-300 g/day); our estimates might be higher, due to the higher availability of fruit and lower prices in the regional territory [34] (taking into account that none of the previous reports included the municipality of Catania for sampling), or represent an overestimation, due to potential limitation of this type of recall studies (i.e., higher number of food items coding for "fruit" compared to other FFQs). Mean vegetable and legume consumption in our study was more in line with worldwide average intake (about $250 \mathrm{~g} /$ day versus $208.8 \mathrm{~g} /$ day, respectively) and those reported in the other Italian studies [32,33]. Moreover, fruit and vegetable consumption were strongly intercorrelated, reflecting a global trend. Consumption of nuts, seeds, and wholegrain is relatively low worldwide (around $10 \mathrm{~g} /$ day and $40 \mathrm{~g} /$ day), with the highest consumption in Southeast Asian nations and the lowest in Central European nations. Our reports were similar to worldwide average regarding whole-grain consumption, but much higher concerning nut intake (about $20 \mathrm{~g} /$ day); again, this can be the result of increased intake due to local production of certain nut subtypes (i.e., pistachios), which might be easier available and at lower price, or an overestimation due to various questions on nut-subtypes in our FFQs. Regarding animal products, in our cohort we found a higher consumption of seafood (about $60 \mathrm{~g} /$ day versus $28 \mathrm{~g}$ /day), similar of processed meat (about $16 \mathrm{~g} /$ day versus $14 \mathrm{~g} /$ day), and slightly lower of unprocessed meat (about $34 \mathrm{~g} /$ day versus $42 \mathrm{~g}$ /day) compared to worldwide reports. However, the higher seafood intake was evident in Pacific Island nations, the Mediterranean Basin, South Korea, and Japan, consistently with historical cultures and local availability. Also, the other Italian report showed similar intake of processed and unprocessed meat products than those reported in the present study, while consumption of fish was lower [32,33]. According to the Italian National Institute of Statistics (ISTAT), the mean expenditure for major food groups in the Italian islands (including Sicily) does not substantially differ from the national average, with the exception of higher purchase of seafood, thus reflecting a regional preference in consuming such products. Interestingly, we have found that seafood intake was weakly correlated with most of the other food groups investigated, suggesting that preference for fish might be common, and related with either healthy or unhealthy food groups.

Global and national reports on macronutrient intake have underlined dramatic diversity across nations and the need for inform policies to improve global health. Our estimates for dietary fats are slightly "healthier" than those previously reported in the Italian population (i.e., lower cholesterol and saturated fatty acid intake) [35]; however, no previous data on specific subgroup of fats (i.e., omega-6, omega-3, etc.) or protein (plant protein, animal protein) has been reported for the Italian population. When comparing our data to global consumption of fat, we reported lower intake of dietary cholesterol 
( $187 \mathrm{mg} /$ day vs. $228 \mathrm{mg}$ /day), higher of seafood omega-3 (0.53 g/day vs. $0.16 \mathrm{~g} /$ day) and similar of plant omega-3 (1.17 g/day vs. $1.37 \mathrm{~g} /$ day) [36]. Comparative data on type of protein is harder to retrieve. By roughly converting our estimates as percentage of total energy $(\% \mathrm{E})$, we may consider that the population investigated in this study consumed an average $5 \% \mathrm{E}$ of animal protein (not including dairy protein) and about $9 \% \mathrm{E}$ of plant protein: cohort studies conducted in the United States reported animal and plant protein intake of about $14 \%$ and $6 \%$, respectively [15]; another Australian cohort reported slightly lower median intake of animal protein (about $10 \%$ of total energy) and similar of plant protein (about 6.5\%) [37,38]. Thus, despite that studies to compare our reports to are scarce, we found a pattern of protein source intake healthier than in the aforementioned countries. These data on macronutrients, together with the aforementioned findings on major food groups, reflects the other findings on adherence to dietary recommendations. Various studies across the globe have reported an overall poor adherence to dietary guidelines of adult populations. Recent reports showed that diet quality of Americans, measured as agreement with dietary recommendations listed in the Healthy Eating Index (HEI), were far from optimal, regardless of socioeconomic status and race. Similarly, comparable trends have been observed in European countries. In Spain, there is a general low adherence to dietary guidelines, and these trends are particularly evident in individuals with overweight and obesity [39]. Nutrition surveys from France [40] and Germany [41] reported that consumption of fruit and vegetable does not meet dietary recommendations: similar findings were showed in other studies, where only about 30\% of people living in United Kingdom [42] and 10\% in Italy [43] reported eating the recommended five portions of fruits and vegetables per day. A report from Eastern European countries showed that roughly half individuals met WHO criteria for fruit and vegetable consumption, but only a minority met those for pulses and nut consumption [44]. By contrast, we found that half to two-thirds of the participants in our cohort met dietary guidelines on macronutrient and food group consumption, with the exception for total fats. However, despite that most of the individuals were under or, most likely, over the recommended intake, the results are not necessarily alarming, as we reported a higher intake of healthy rather than unhealthy fats. It has been shown that food sources of fat, such as olive oil, fish, and nuts, are associated with positive outcomes for health and a general recommendation in limiting total dietary fats may not entirely reflect a proper advice [45-47].

In this study, we found a correlation between certain macronutrients and food groups with BMI levels of the participants. Mostly in line with expectations, among dietary proteins, only plant protein intake was inversely correlated with BMI, while among dietary fats, monounsaturated and omega- 6 fatty acids were inversely correlated, whereas trans fatty acids and cholesterol were directly correlated. However, these results did not entirely fit with correlations obtained with major food groups, as processed and unprocessed meats were indirectly and directly correlated with BMI levels, respectively. A possible reason for such unexpected findings may be the relative good quality of processed meat in southern Italy, which according the results of individual questions of the FFQ, we found it mostly referred to cured meat rather than fast foods (data not shown). Another explanation is the relatively low magnitude of the correlation for protein and meat products, which in fact might be spurious. Regarding the findings on dietary fats, we hypothesize that a major contributor to monounsaturated fatty acid intake was olive oil, highly consumed in this cohort as reported in previous studies [48]. General high levels of adherence to the Mediterranean diet has been previously shown in this cohort, as well as the association with lower likelihood of being obese and other metabolic conditions for those participants highly adherent to this dietary pattern; however, the association was not driven by olive oil or any other of the components of the score [49-51]. These findings corroborate the results of several other studies and suggest that the overall dietary pattern was more descriptive for a healthy nutritional alternative associated with better metabolic health [52-54]. Possible mediating factors have been hypothesized to be dietary polyphenols, which have been reported to exert potential beneficial effects on health $[55,56]$. With special regards to metabolic outcomes, dietary polyphenols have been shown to mediate, at least in part, the observed association with better metabolic health in 
this cohort [57-59]. Further studies are needed to investigate whether such compounds may explain, from a mechanistic point of view, the beneficial effects of healthy dietary pattern rich in fruit and vegetable, and other features typical of the Mediterranean diet.

The results presented in this study should be considered in light of methodological limitations. The use of FFQs is a widely-consolidated methodology, but they are also known to only provide estimates and not true intake, as they are subject to recall bias and over- and underestimation, depending on the number of food items included and social desirability bias, respectively. However, comparative reports used similar methodology and results are generally in line with literature and expected findings.

\section{Conclusions}

In conclusion, the present study provided updated information on macronutrient and major food group intake in a southern Italian adult population, taking into account specific subgroup of macronutrients rarely reported in current literature. The overall findings suggest that relatively healthy dietary habits are common in southern Italy, in up to two-thirds of the sample investigated. Further in-depth studies are needed to better understand whether findings related to foods may translate in equally adequate micronutrient intake in this cohort. However, further efforts should be made to improve diet quality of the remaining population in order to prevent non-communicable diseases.

Author Contributions: M.M. conceived and designed the experiments; A.P. performed the experiments; M.F., A.C., D.C. and I.B. analyzed the data; S.M. and M.M. and M.D'U. wrote the paper.

Conflicts of Interest: The authors declare no conflict of interest.

\section{References}

1. Lim, S.S.; Vos, T.; Flaxman, A.D.; Danaei, G.; Shibuya, K.; Adair-Rohani, H.; Amann, M.; Anderson, H.R.; Andrews, K.G.; Aryee, M.; et al. A comparative risk assessment of burden of disease and injury attributable to 67 risk factors and risk factor clusters in 21 regions, 1990-2010: A systematic analysis for the global burden of disease study 2010. Lancet 2012, 380, 2224-2260. [CrossRef]

2. Grosso, G.; Bella, F.; Godos, J.; Sciacca, S.; Del Rio, D.; Ray, S.; Galvano, F.; Giovannucci, E.L. Possible role of diet in cancer: Systematic review and multiple meta-analyses of dietary patterns, lifestyle factors, and cancer risk. Nutr. Rev. 2017, 75, 405-419. [CrossRef] [PubMed]

3. Mozaffarian, D. Dietary and policy priorities for cardiovascular disease, diabetes, and obesity: A comprehensive review. Circulation 2016, 133, 187-225. [CrossRef] [PubMed]

4. Khatibzadeh, S.; Saheb Kashaf, M.; Micha, R.; Fahimi, S.; Shi, P.; Elmadfa, I.; Kalantarian, S.; Wirojratana, P.; Ezzati, M.; Powles, J.; et al. A global database of food and nutrient consumption. Bull. World Health Organ. 2016, 94, 931-934. [CrossRef] [PubMed]

5. Morgan, P.J. Back to the future: The changing frontiers of nutrition research and its relationship to policy. Proc. Nutr. Soc. 2012, 71, 190-197. [CrossRef] [PubMed]

6. Mozaffarian, D.; Ludwig, D.S. Dietary guidelines in the 21st century-A time for food. JAMA 2010, 304, 681-682. [CrossRef] [PubMed]

7. Ezzati, M.; Riboli, E. Behavioral and dietary risk factors for noncommunicable diseases. N. Engl. J. Med. 2013, 369, 954-964. [CrossRef] [PubMed]

8. Nishida, C.; Uauy, R.; Kumanyika, S.; Shetty, P. The joint WHO/FAO expert consultation on diet, nutrition and the prevention of chronic diseases: Process, product and policy implications. Public Health Nutr. 2004, 7, 245-250. [CrossRef] [PubMed]

9. Mozaffarian, D. Foods, obesity, and diabetes-are all calories created equal? Nutr. Rev. 2017, 75, 19-31. [CrossRef] [PubMed]

10. Jebb, S.A. Carbohydrates and obesity: From evidence to policy in the UK. Proc. Nutr. Soc. 2015, 74, $215-220$. [CrossRef] [PubMed] 
11. Santesso, N.; Akl, E.A.; Bianchi, M.; Mente, A.; Mustafa, R.; Heels-Ansdell, D.; Schunemann, H.J. Effects of higher-versus lower-protein diets on health outcomes: A systematic review and meta-analysis. Eur. J. Clin. Nutr. 2012, 66, 780-788. [CrossRef] [PubMed]

12. Wycherley, T.P.; Moran, L.J.; Clifton, P.M.; Noakes, M.; Brinkworth, G.D. Effects of energy-restricted high-protein, low-fat compared with standard-protein, low-fat diets: A meta-analysis of randomized controlled trials. Am. J. Clin. Nutr. 2012, 96, 1281-1298. [CrossRef] [PubMed]

13. Tian, S.; Xu, Q.; Jiang, R.; Han, T.; Sun, C.; Na, L. Dietary protein consumption and the risk of type 2 diabetes: A systematic review and meta-analysis of cohort studies. Nutrients 2017, 9, 982. [CrossRef] [PubMed]

14. Farvid, M.S.; Malekshah, A.F.; Pourshams, A.; Poustchi, H.; Sepanlou, S.G.; Sharafkhah, M.; Khoshnia, M.; Farvid, M.; Abnet, C.C.; Kamangar, F.; et al. Dietary protein sources and all-cause and cause-specific mortality: The golestan cohort study in Iran. Am. J. Prev. Med. 2017, 52, 237-248. [CrossRef] [PubMed]

15. Song, M.; Fung, T.T.; Hu, F.B.; Willett, W.C.; Longo, V.D.; Chan, A.T.; Giovannucci, E.L. Association of animal and plant protein intake with all-cause and cause-specific mortality. JAMA Intern. Med. 2016, 176, 1453-1463. [CrossRef] [PubMed]

16. Dalen, J.E.; Devries, S. Diets to prevent coronary heart disease 1957-2013: What have we learned? Am. J. Med. 2014, 127, 364-369. [CrossRef] [PubMed]

17. Bloomfield, H.E.; Koeller, E.; Greer, N.; MacDonald, R.; Kane, R.; Wilt, T.J. Effects on health outcomes of a mediterranean diet with no restriction on fat intake: A systematic review and meta-analysis. Ann. Intern. Med. 2016, 165, 491-500. [CrossRef] [PubMed]

18. Grosso, G.; Marventano, S.; Yang, J.; Micek, A.; Pajak, A.; Scalfi, L.; Galvano, F.; Kales, S.N. A comprehensive meta-analysis on evidence of mediterranean diet and cardiovascular disease: Are individual components equal? Crit. Rev. Food Sci. Nutr. 2017, 57, 3218-3232. [CrossRef] [PubMed]

19. Liu, A.G.; Ford, N.A.; Hu, F.B.; Zelman, K.M.; Mozaffarian, D.; Kris-Etherton, P.M. A healthy approach to dietary fats: Understanding the science and taking action to reduce consumer confusion. Nutr. J. 2017, 16, 53. [CrossRef] [PubMed]

20. Marventano, S.; Kolacz, P.; Castellano, S.; Galvano, F.; Buscemi, S.; Mistretta, A.; Grosso, G. A review of recent evidence in human studies of n-3 and n-6 PUFA intake on cardiovascular disease, cancer, and depressive disorders: Does the ratio really matter? Int. J. Food Sci. Nutr. 2015, 66, 611-622. [CrossRef] [PubMed]

21. Grosso, G.; Marventano, S.; D’Urso, M.; Mistretta, A.; Galvano, F. The mediterranean healthy eating, ageing, and lifestyle (MEAL) study: Rationale and study design. Int. J. Food Sci. Nutr. 2017, 68, 577-586. [CrossRef] [PubMed]

22. Mistretta, A.; Marventano, S.; Platania, A.; Godos, J.; Galvano, F.; Grosso, G. Metabolic profile of the mediterranean healthy eating, lifestyle and aging (MEAL) study cohort. Mediterr. J. Nutr. Metab. 2017, 10, 131-140. [CrossRef]

23. World Health Organization. Obesity: Preventing and Managing the Global Epidemic; Report of a Who Consultation Presented at the World Health Organization, 3-5 June 1997; World Health Organization: Geneva, Switzerland, 1997.

24. Buscemi, S.; Rosafio, G.; Vasto, S.; Massenti, F.M.; Grosso, G.; Galvano, F.; Rini, N.; Barile, A.M.; Maniaci, V.; Cosentino, L.; et al. Validation of a food frequency questionnaire for use in Italian adults living in sicily. Int. J. Food Sci. Nutr. 2015, 66, 426-438. [CrossRef] [PubMed]

25. Marventano, S.; Mistretta, A.; Platania, A.; Galvano, F.; Grosso, G. Reliability and relative validity of a food frequency questionnaire for Italian adults living in Sicily, Southern Italy. Int. J. Food Sci. Nutr. 2016, 67, 857-864. [CrossRef] [PubMed]

26. Istituto Nazionale di Ricerca per gli Alimenti e la Nutrizione. Tabelle di Composizione Degli Alimenti; Istituto Nazionale di Ricerca per gli Alimenti e la Nutrizione: Rome, Italy, 2009.

27. Willett, W.C.; Lenart, E. Reproducibility and validity of food frequency questionnaire. In Nutritional Epidemiology, 2nd ed.; Oxford University Press: Oxford, UK, 1998.

28. EFSA. Dietary Reference Values for Nutrients. Available online: https:/ / www.efsa.europa.eu/sites/default/ files/2017_09_DRVs_summary_report.pdf (accessed on 18 March 2018).

29. Società Italiana di Nutrizione Umana (SINU). Iv Revisione dei Livelli di Assunzione di Riferimento di Nutrienti ed Energia per la Popolazione Italiana (LARN). Available online: http:/ / www.sinu.it/html/pag/ tabelle_larn_2014_rev.asp (accessed on 18 Marh 2018). 
30. WHO. Diet, Nutrition and the Prevention of Chronic Diseases. Available online: http:/ / apps.who.int/iris/bitstream/handle/10665/42665/WHO_TRS_916.pdf;jsessionid= B7E43124EB39AE47E19C06B0A876C0FD?sequence=1 (accessed on 18 March 2018).

31. Micha, R.; Khatibzadeh, S.; Shi, P.; Andrews, K.G.; Engell, R.E.; Mozaffarian, D.; Global Burden of Diseases Nutrition; Chronic Diseases Expert Group. Global, regional and national consumption of major food groups in 1990 and 2010: A systematic analysis including 266 country-specific nutrition surveys worldwide. BMJ Open 2015, 5, e008705. [CrossRef] [PubMed]

32. Pounis, G.; Bonanni, A.; Ruggiero, E.; Di Castelnuovo, A.; Costanzo, S.; Persichillo, M.; Bonaccio, M.; Cerletti, C.; Riccardi, G.; Donati, M.B.; et al. Food group consumption in an Italian population using the updated food classification system FoodEx2: Results from the Italian Nutrition \& Health Survey (INHES) study. Nutr. Metab. Cardiovasc. Dis. 2017, 27, 307-328. [CrossRef] [PubMed]

33. Leclercq, C.; Arcella, D.; Piccinelli, R.; Sette, S.; Le Donne, C.; Turrini, A.; INRAN-SCAI 2005-06 Study Group. The Italian national food consumption survey INRAN-SCAI 2005-06: Main results in terms of food consumption. Public Health Nutr. 2009, 12, 2504-2532. [CrossRef] [PubMed]

34. EUROSTAT. Comparative Price Levels for Food, Beverages and Tobacco. Available online: http:/ / ec.europa.eu/eurostat/statistics-explained/index.php/Comparative_price_levels_for_food,_beverages_ and_tobacco-Bread_and_cereals.2C_meat.2C_fish_and_dairy_products (accessed on 26 January 2018).

35. Sette, S.; Le Donne, C.; Piccinelli, R.; Arcella, D.; Turrini, A.; Leclercq, C.; Group, I.-S.S. The third Italian national food consumption survey, INRAN-SCAI 2005-06-Part 1: Nutrient intakes in Italy. Nutr. Metab. Cardiovasc. Dis. 2011, 21, 922-932. [CrossRef] [PubMed]

36. Micha, R.; Khatibzadeh, S.; Shi, P.; Fahimi, S.; Lim, S.; Andrews, K.G.; Engell, R.E.; Powles, J.; Ezzati, M.; Mozaffarian, D.; et al. Global, regional, and national consumption levels of dietary fats and oils in 1990 and 2010: A systematic analysis including 266 country-specific nutrition surveys. BMJ 2014, 348, g2272. [CrossRef] [PubMed]

37. Shang, X.; Scott, D.; Hodge, A.; English, D.R.; Giles, G.G.; Ebeling, P.R.; Sanders, K.M. Dietary protein from different food sources, incident metabolic syndrome and changes in its components: An 11-year longitudinal study in healthy community-dwelling adults. Clin. Nutr. 2017, 36, 1540-1548. [CrossRef] [PubMed]

38. Shang, X.; Scott, D.; Hodge, A.M.; English, D.R.; Giles, G.G.; Ebeling, P.R.; Sanders, K.M. Dietary protein intake and risk of type 2 diabetes: Results from the melbourne collaborative cohort study and a meta-analysis of prospective studies. Am. J. Clin. Nutr. 2016, 104, 1352-1365. [CrossRef] [PubMed]

39. Rodriguez-Rodriguez, E.; Aparicio, A.; Aranceta-Bartrina, J.; Gil, A.; Gonzalez-Gross, M.; Serra-Majem, L.; Varela-Moreiras, G.; Ortega, R.M. Low adherence to dietary guidelines in Spain, especially in the overweight/obese population: The ANIBES study. J. Am. Coll. Nutr. 2017, 36, 240-247. [CrossRef] [PubMed]

40. Castetbon, K.; Vernay, M.; Malon, A.; Salanave, B.; Deschamps, V.; Roudier, C.; Oleko, A.; Szego, E.; Hercberg, S. Dietary intake, physical activity and nutritional status in adults: The French nutrition and health survey (ENNS, 2006-2007). Br. J. Nutr. 2009, 102, 733-743. [CrossRef] [PubMed]

41. Gose, M.; Krems, C.; Heuer, T.; Hoffmann, I. Trends in food consumption and nutrient intake in Germany between 2006 and 2012: Results of the german national nutrition monitoring (NEMONIT). Br. J. Nutr. 2016, 115, 1498-1507. [CrossRef] [PubMed]

42. Whitton, C.; Nicholson, S.K.; Roberts, C.; Prynne, C.J.; Pot, G.K.; Olson, A.; Fitt, E.; Cole, D.; Teucher, B.; Bates, B.; et al. National diet and nutrition survey: UK food consumption and nutrient intakes from the first year of the rolling programme and comparisons with previous surveys. Br. J. Nutr. 2011, 106, 1899-1914. [CrossRef] [PubMed]

43. Istituto Superiore di Sanità. Rapporto Nazionale Passi 2012: Consumo di Frutta e Verdura. Available online: http:/ / www.epicentro.iss.it/passi/rapporto2012/5ADay.asp (accessed on 23 February 2018).

44. Boylan, S.; Welch, A.; Pikhart, H.; Malyutina, S.; Pajak, A.; Kubinova, R.; Bragina, O.; Simonova, G.; Stepaniak, U.; Gilis-Januszewska, A.; et al. Dietary habits in three central and eastern european countries: The hapiee study. BMC Public Health 2009, 9, 439. [CrossRef] [PubMed]

45. Grosso, G.; Estruch, R. Nut consumption and age-related disease. Maturitas 2016, 84, 11-16. [CrossRef] [PubMed] 
46. Lopez-Miranda, J.; Perez-Jimenez, F.; Ros, E.; De Caterina, R.; Badimon, L.; Covas, M.I.; Escrich, E.; Ordovas, J.M.; Soriguer, F.; Abia, R.; et al. Olive oil and health: Summary of the II international conference on olive oil and health consensus report, Jaen and Cordoba (Spain) 2008. Nutr. Metab. Cardiovasc. Dis. 2010, 20, 284-294. [CrossRef] [PubMed]

47. Mozaffarian, D.; Rimm, E.B. Fish intake, contaminants, and human health: Evaluating the risks and the benefits. JAMA 2006, 296, 1885-1899. [CrossRef] [PubMed]

48. Marventano, S.; Godos, J.; Platania, A.; Galvano, F.; Mistretta, A.; Grosso, G. Mediterranean diet adherence in the mediterranean healthy eating, aging and lifestyle (MEAL) study cohort. Int. J. Food Sci. Nutr. 2018, 69, 100-107. [CrossRef] [PubMed]

49. Zappala, G.; Buscemi, S.; Mule, S.; La Verde, M.; D’Urso, M.; Corleo, D.; Marranzano, M. High adherence to mediterranean diet, but not individual foods or nutrients, is associated with lower likelihood of being obese in a mediterranean cohort. Eat. Weight Disord. 2017, 1-10. [CrossRef] [PubMed]

50. La Verde, M.; Mule, S.; Zappala, G.; Privitera, G.; Maugeri, G.; Pecora, F.; Marranzano, M. Higher adherence to the mediterranean diet is inversely associated with having hypertension: Is low salt intake a mediating factor? Int. J. Food Sci. Nutr. 2018, 69, 235-244. [CrossRef] [PubMed]

51. Platania, A.; Zappala, G.; Mirabella, M.U.; Gullo, C.; Mellini, G.; Beneventano, G.; Maugeri, G.; Marranzano, M. Association between mediterranean diet adherence and dyslipidaemia in a cohort of adults living in the Mediterranean area. Int. J. Food Sci. Nutr. 2017, 1-11. [CrossRef] [PubMed]

52. Mancini, J.G.; Filion, K.B.; Atallah, R.; Eisenberg, M.J. Systematic review of the mediterranean diet for long-term weight loss. Am. J. Med. 2016, 129, 407-415.e4. [CrossRef] [PubMed]

53. Grosso, G.; Mistretta, A.; Marventano, S.; Purrello, A.; Vitaglione, P.; Calabrese, G.; Drago, F.; Galvano, F. Beneficial effects of the mediterranean diet on metabolic syndrome. Curr. Pharm. Des. 2014, 20, 5039-5044. [CrossRef] [PubMed]

54. Godos, J.; Zappala, G.; Bernardini, S.; Giambini, I.; Bes-Rastrollo, M.; Martinez-Gonzalez, M. Adherence to the mediterranean diet is inversely associated with metabolic syndrome occurrence: A meta-analysis of observational studies. Int. J. Food Sci. Nutr. 2017, 68, 138-148. [CrossRef] [PubMed]

55. Grosso, G.; Godos, J.; Lamuela-Raventos, R.; Ray, S.; Micek, A.; Pajak, A.; Sciacca, S.; D’Orazio, N.; Del Rio, D.; Galvano, F. A comprehensive meta-analysis on dietary flavonoid and lignan intake and cancer risk: Level of evidence and limitations. Mol. Nutr. Food Res. 2017, 61. [CrossRef] [PubMed]

56. Grosso, G.; Micek, A.; Godos, J.; Pajak, A.; Sciacca, S.; Galvano, F.; Giovannucci, E.L. Dietary flavonoid and lignan intake and mortality in prospective cohort studies: Systematic review and dose-response meta-analysis. Am. J. Epidemiol. 2017, 185, 1304-1316. [CrossRef] [PubMed]

57. Godos, J.; Bergante, S.; Satriano, A.; Pluchinotta, F.R.; Marranzano, M. Dietary phytoestrogen intake is inversely associated with hypertension in a cohort of adults living in the Mediterranean area. Molecules 2018, 23, 368. [CrossRef] [PubMed]

58. Godos, J.; Sinatra, D.; Blanco, I.; Mule, S.; La Verde, M.; Marranzano, M. Association between dietary phenolic acids and hypertension in a mediterranean cohort. Nutrients 2017, 9, 69. [CrossRef]

59. Marranzano, M.; Ray, S.; Godos, J.; Galvano, F. Association between dietary flavonoids intake and obesity in a cohort of adults living in the Mediterranean area. Int. J. Food Sci. Nutr. 2018, 1-10. [CrossRef] [PubMed]

(C) 2018 by the authors. Licensee MDPI, Basel, Switzerland. This article is an open access article distributed under the terms and conditions of the Creative Commons Attribution (CC BY) license (http://creativecommons.org/licenses/by/4.0/). 